\title{
Iron Oxide-based Superparamagnetic Polymeric Nanomaterials: Design, Preparation, and Biomedical Application
}

Jung Kwon $\mathrm{Oh}^{*}$

Department of Chemistry and Biochemistry, Concordia University, 7141 Sherbrooke Street West, Montreal, Quebec, Canada H4B 1R6

Tel: 1-514-848-2424, email: joh@alcor.concordia.ca

Jong Myung Park ${ }^{*}$

Surface Engineering Laboratory, Graduate Institute of Ferrous Technology, Pohang University of Science and Technology, Pohang 790-784, Korea

Email:jongpark@postech.ac.kr 


\begin{abstract}
Superparamagnetic iron oxide nanoparticles (SIONPs) have great potential for various biomedical applications, including magnetic resonance imaging (MRI) contrast enhancement, targeted drug delivery, hyperthermia, catalysis, biological separation, biosensors, and diagnostic medical devices. For the development of SIONPs toward bio-related applications, control of the surface chemistry of SIONPs is required. Polymers with more than one group capable of binding to particle surfaces (multidentate ligands) can enhance the stability of SIONPs as well as their optical, magnetic, and electronic properties. Most synthetic and bio-based polymers are transparent in the visible range of electromagnetic spectrum, not interfering with biological process. Additionally, polymers provide mechanical and chemical stability to the nanomaterials. The present review summarizes the recent advances in design and biological applications of polymer-embedded SIONPs.
\end{abstract}

\title{
Outline
}

\section{Introduction}

1.1. Synthesis of superparamagnetic iron oxide NPs (SIONPs)

2. Direct modification with polymers

3. Surface-initiated controlled polymerization: "grafting from" method

4. Inorganic silica/polymer hybridization

5. Self-assembly and self-association

6. Heterogeneous polymerization

6.1. Inverse (mini)emulsion polymerization

6.2. Dispersion polymerization

6.3. Other heterogeneous polymerization methods

7. Bulk physical and chemical crosslinking-magnetic hydrogel preparation

8. Bio-applications of SIONP-polymer hybrids

8.1. MR imaging

8.2. Drug delivery

8.3. Other applications including hyperthermia, protein immobilization, and catalysts

9. Conclusion

10. References 


\section{Introduction}

Colloidal inorganic nanometer-sized particles (nanoparticles, NPs) or nanocrystals (NCs) have proved to be useful as building blocks for the development of nanomaterials and biomaterials in nanoscience and biotechnology. This is because of their unique structural and optical properties that are attributed to nanoscale phenomena. ${ }^{[1]}$ Superparamagnetic iron oxide NPs (SIONPs) including $\mathrm{Fe}_{3} \mathrm{O}_{4}$ magnetite and $\mathrm{Fe}_{2} \mathrm{O}_{3}$ maghemite have great potential for various biomedical applications. They include magnetic resonance imaging (MRI) contrast enhancement, targeted drug delivery, hyperthermia, catalysis, biological separation, biosensors, and diagnostic medical devices. ${ }^{[2-8]}$ Other magnetic NPs have also been developed, including iron-based $\mathrm{FePt},{ }^{[9-15]} \mathrm{FePd},{ }^{[16]} \mathrm{Co}$-based $\mathrm{CoPt},{ }^{[17,18]} \mathrm{CoO},{ }^{[19]}$ and $\mathrm{CoFe}_{2} \mathrm{O}_{4},{ }^{[20]}$ as well as Mn-based $\mathrm{MnPt},{ }^{[21]} \mathrm{Gd}-$ based NPs, ${ }^{[22-26]}$ and their inorganic-inorganic hybrid nanomaterials. ${ }^{[27-33]}$

For the development of SIONPs toward bio-related applications, control of the surface chemistry of SIONPs is required. Pristine SIONPs tend to aggregate into large clusters, because of their large surface-to-volume ratio and dipole-dipole interaction. The resulting large agglomerates reduce intrinsic superparamagnetic properties. The surface of SIONPs has been modified to not only prevent aggregation of the particles, leading to colloidal stability, but also render them with water-solubility, biocompatibility, and nonspecific adsorption to cells. In addition, control of surface chemistry can allow for the flexibility and functionality of SIONPs that enable efficient coupling of these probes to bioactive molecules capable of targeting and sensing biological processes. Several approaches to modification of the surface of SIONPs with small molecules including biomolecules have been investigated for the preparation of water-soluble SIONPs. The general approach is the post-addition of water-soluble ligands, including direct adsorption, ${ }^{[34-38]}$ addition of second layer, ${ }^{[39-41]}$ ligand exchange, ${ }^{[23,42-45]}$ functional silica coating, ${ }^{[46-50]}$ and ionic interaction. ${ }^{[51]}$ In-situ formation approach directly yields water-soluble SIONPs 
in the presence of stabilizing ligands. Typical examples include $\mathrm{Fe}_{3} \mathrm{O}_{4}$ NPs coated with D-mannose, ${ }^{[52]}$ 2-pyrrolidone, ${ }^{[53]}$ and poly(ethylene glycol) diacids (HOOC-PEG-COOH), ${ }^{[54]}$ as well as iron oxide nanoworms coated with dextran (Dex). ${ }^{[55]}$

Polymers with more than one group capable of binding to particle surfaces (multidentate ligands) can enhance colloidal stability of inorganic NPs including SIONPs as well as their optical, magnetic, and electronic properties. ${ }^{[56]}$ Most synthetic and bio-based polymers are transparent in the visible range of electromagnetic spectrum, not interfering with biological process. Additionally, polymers provide mechanical and chemical stability to the nanomaterials. The present review will summarize the recent advances in design, preparation, and biological application of polymer-embedded SIONPs. The methods that have been developed to prepare unique polymer-SIONP hybrid nanomaterials include direct modification with polymers, surface-initiated controlled polymerization, inorganic silica/polymer hybridization, self-assembly, self-association, and various heterogeneous polymerization methods. These methods provide magnetic polymer composites that differ in morphologies. ${ }^{[57]}$ Figure 1 illustrates the various morphologies, including magnetic core-polymer shell (a), magnetic multicores homogeneously dispersed in polymer matrix (b), magnetic NPs located on the surface of a polymer core ("raspberry" morphology, c), and brush (hair)-like morphology with polymer chains attached to a magnetic core $(d)$. 

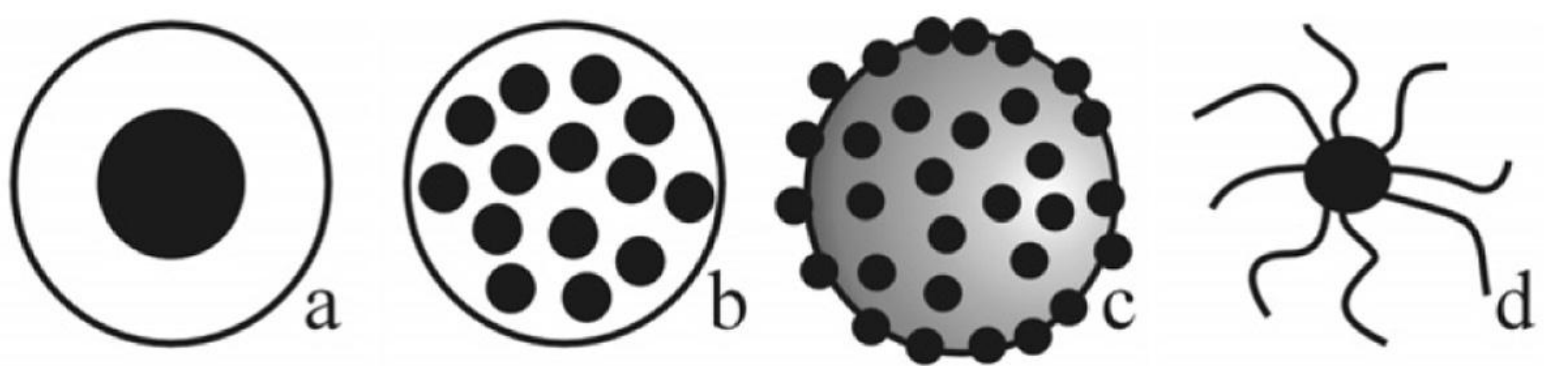

Figure 1. Different morphologies of composite magnetic polymer microspheres; single-core (a), multicore or embedded (b), raspberry-like or heterocoagulated (c), and brush-like morphology (d). Reprinted with permission from ref ${ }^{[57]}$. Copyright 2007 Wiley InterScience.

\subsection{Synthesis of superparamagnetic iron oxide NPs (SIONPs)}

Coprecipitation of $\mathrm{Fe}(\mathrm{II})$ and $\mathrm{Fe}(\mathrm{III})$ ions from an aqueous basic solution is a facile method for the preparation of SIONPs in water. Several parameters should be controlled to prepare SIONPs with narrow size distribution. They include $\mathrm{pH}$, temperature, and mixing method, as well as nature and concentration of anions. In general, $\mathrm{FeCl}_{3}$ and $\mathrm{FeCl}_{2}$ solutions are mixed at a concentration ratio of $\mathrm{Fe}(\mathrm{III}) / \mathrm{Fe}(\mathrm{II})=2 / 1$ in an aqueous ammonia solution, yielding $\mathrm{Fe}_{3} \mathrm{O}_{4}$ SIONPs with $\mathrm{d}=3-15 \mathrm{~nm}$. Recently, a droplet-based microfluidic system has been designed to prepare SIONPs via coprecipitation of $\mathrm{Fe}(\mathrm{II})$ and $\mathrm{Fe}(\mathrm{III})$ solutions in an continuous oil phase (Figure 2). The microfluidic device was designed in such by injecting two aqueous Fe salt solutions through the outer channels, which were synchronously emulsified by central oil channel. This approach enabled fast (millisecond scale) preparation of SIONPs with $d=4 n$ m. ${ }^{[58]}$ 


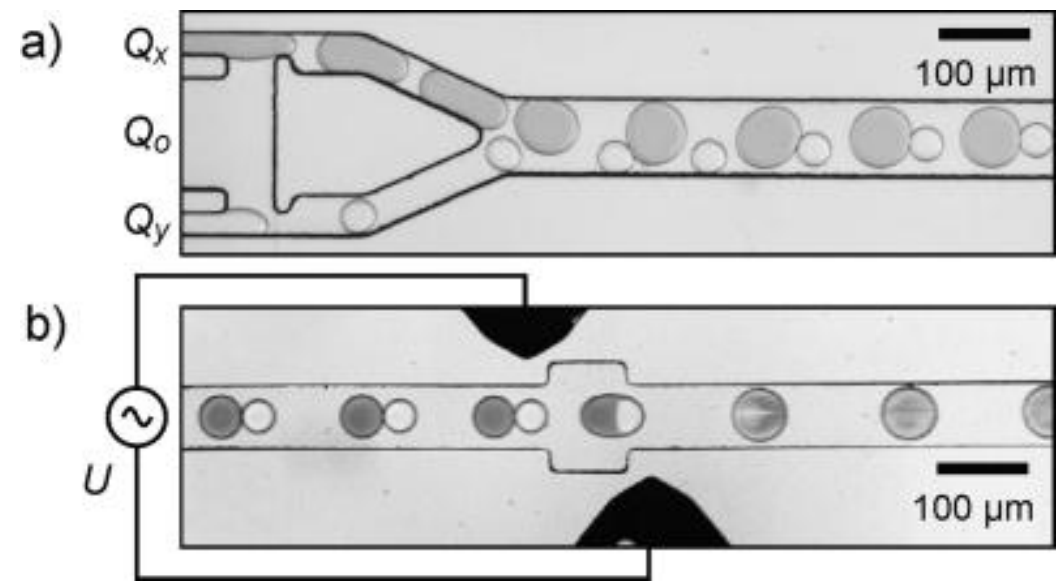

Figure 2. Design of droplet-based microfluidic system for the preparation of magnetic $\mathrm{Fe}_{3} \mathrm{O}_{4}$ nanocrystals via coprecipitation of aqueous $\mathrm{Fe}(\mathrm{II})$ and $\mathrm{Fe}$ (III) solutions in oil. Pairing module with $Q_{0}$ for oil and $Q_{x}$ and $Q_{y}$ for two aqueous phases (a); fusion module where paired droplets are coalesced by applying an electric voltage $U$ between two electrodes (b). Reprinted with permission from ref ${ }^{[58]}$. Copyright 2008 Wiley InterScience.

Organic solution-phase synthesis, also known as thermal decomposition of iron precursor in hot surfactant solutions, has been developed for the preparation of high-quality monodisperse SIONPs. Direct decomposition of $\mathrm{FeCup}_{3}{ }^{[59]}$ and $\mathrm{Fe}(\mathrm{CO})_{5}{ }^{[60]}$ followed by oxidation produced monodisperse $\mathrm{Fe}_{2} \mathrm{O}_{3}$ maghemite nanocrystals. High temperature reaction of $\mathrm{Fe}(\mathrm{III})$ acetylacetonate $\left(\mathrm{Fe}(\mathrm{acac})_{3}\right)$ in the presence of oleic acid and oleylamine as stabilizing ligands in phenyl ether at $265^{\circ} \mathrm{C}$ yielded monodisperse $\mathrm{Fe}_{3} \mathrm{O}_{4}$ magnetite NPs with a diameter of $16 \mathrm{~nm}$. The resulting magnetite NPs was oxidated to $\mathrm{Fe}_{2} \mathrm{O}_{3}$ maghemite NPs at $250{ }^{\circ} \mathrm{C}$ in the presence of oxygen for $2 \mathrm{~h} \cdot{ }^{[61]} \mathrm{A}$ mild condition of thermal decomposition of $\mathrm{Fe}(\mathrm{CO})_{5}$ in oleic acid and octyl ether at $100{ }^{\circ} \mathrm{C}$ for $2 \mathrm{~h}$ and consecutive aeration was developed for the preparation of maghemite NPs with a diameter ranging from 5 to $19 \mathrm{~nm}$ and magnetite NPs with $19 \mathrm{~nm}$ diameter, as seen in TEM images (Figure 3) ${ }^{[62]}$ Recently, thermal decomposition of iron oleate precursors in the presence of oleic acid salts was reported for the preparation of iron oxide nanocrystals with various shapes including spheres, cubes, and bipyrimids. ${ }^{[63]}$ In addition, the thermal decomposition method combined with thermal oxidation was explored for the preparation of hollow $\mathrm{Fe}_{3} \mathrm{O}_{4}$ from $\mathrm{Fe} / \mathrm{Fe}_{3} \mathrm{O}_{4}$ core/shell NPs. ${ }^{[64]}$ To avoid the use of oleylamine, oleic acid, 
or trioctylamine which may create environmental concerns, the thermal decomposition of $\mathrm{Fe}(\mathrm{acac})_{3}$ in the presence of environmentally friendly benzyl alcohol at $200{ }^{\circ} \mathrm{C}$ for 2 days was conducted, producing $\mathrm{Fe}_{3} \mathrm{O}_{4}$ SIONPs with a diameter ranging from 12 to $25 \mathrm{~nm} .{ }^{[65]}$
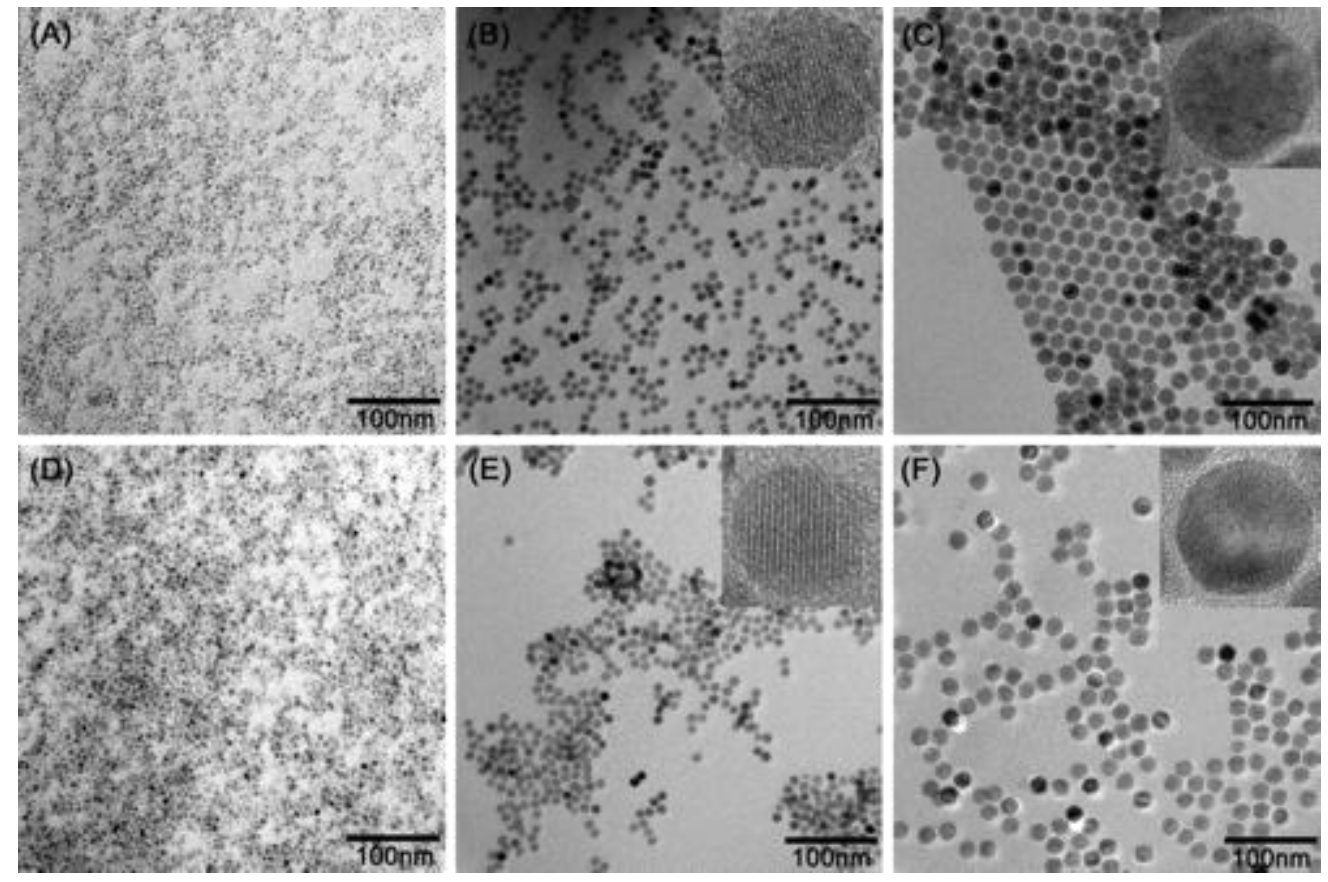

Figure 3. TEM images of respective intermediate and aerated iron oxide NPs: $5 \mathrm{~nm}(\mathrm{~A})$ and $(D), 11 \mathrm{~nm}(B)$ and $(E)$, and $19 \mathrm{~nm}(C)$ and $(F)$; insets are High resolution (HR) TEM images. Reprinted with permission from ref ${ }^{[62]}$. Copyright 2004 American Chemical Society.

Solvothermal reaction by reduction of $\mathrm{FeCl}_{3}$ in hot organic solvents such as ethylene glycol has been explored for the preparation of water-dispersible $\mathrm{Fe}_{3} \mathrm{O}_{4}$ ferrite microspheres, ${ }^{[66-68]}$ microclusters, ${ }^{[69]}$ and nanorings ${ }^{[70]}$ with a diameter of $30-800 \mathrm{~nm}$. In addition, hetero-structured nanocrystals (or heterogeneous inorganic-inorganic hybrid NPs) containing iron oxides have been explored to integrate multiple nanocrystal components into a single nanosystem. Examples include $\mathrm{Fe}_{2} \mathrm{O}_{3} / \mathrm{ZnS}^{[71]}$ and $\mathrm{Fe}_{3} \mathrm{O}_{4} / \mathrm{Au}^{[72,73]}$ core/shell, $\mathrm{FePt} / \mathrm{Fe}_{2} \mathrm{O}_{3}$ yolk-shell, ${ }^{[74]}$ as well as $\mathrm{Fe}_{3} \mathrm{O}_{4} / \mathrm{CdSe}^{[75,76]}$ heterodimers, and $\mathrm{Fe}_{3} \mathrm{O}_{4}$ core/layered double hydroxide. ${ }^{[77]} \mathrm{Mn}$-doped iron oxide nanoparticles were prepared to enhance 
MRI contrast and hyperthermic effects. ${ }^{[78-81]}$ In addition, the preparation of various silica-coated $\mathrm{Fe}_{2} \mathrm{O}_{3}$ or $\mathrm{Fe}_{3} \mathrm{O}_{4} \mathrm{NPs},{ }^{[82-85]}$ silica/ $\mathrm{Fe}_{3} \mathrm{O}_{4}$ core/shell, ${ }^{[86]} \mathrm{Fe}_{2} \mathrm{O}_{3} / \mathrm{SiO}_{2}$ Janus, ${ }^{[87]}$ and silica-satellite $\mathrm{Fe}_{3} \mathrm{O}_{4} \mathrm{NPs}^{[88]}$ has been reported.

\section{Direct modification with polymers}

Various approaches to modify SIONPs with multidentate polymers (possessing multiple anchoring groups in polymer backbones) have been explored to prepare water-soluble/water-dispersible polymerstabilized SIONPs. The typical approaches include physical adsorption, addition of second layer, functional silica coating, and ionic interaction. For the approaches, novel polymers including synthetic polymers and biopolymers are designed, prepared, modified by various methods.

The physical adsorption can be achieved by addition of stabilizing copolymers either during or after the preparation of SIONPs. This approach includes the design and preparation of functional copolymers with two different blocks. An anchoring block contains particularly carboxylic acids that enable the adhesion to the surface of SIONPs; another water-soluble block contains water-soluble groups, particularly poly(ethylene oxide) (PEO), that render SIONPs water-soluble and biocompatible. Control radical polymerization $(\mathrm{CRP})^{[89]}$ methods have been utilized to prepare well-controlled block copolymers with predetermined molecular weight and narrow molecular weight distribution $\left(\mathrm{M}_{\mathrm{w}} / \mathrm{M}_{\mathrm{n}}<\right.$ 1.3). Atom transfer radical polymerization (ATRP) has allowed for the preparation of well-controlled poly(oligo(ethylene oxide) monomethyl ether methacrylate)-b-poly(t-butyl acrylate) (POEOMA-bPtBA) block copolymer. The resulting POEOMA-b-PtBA was then hydrolyzed in acidic conditions, yielding POEOMA-b-poly(methacrylic acid) (POEOMA-b-PMAA). PMAA block is anchored to SIONP surface and POEOMA block renders water-soluble and biocompatible. In the presence of watersoluble POEOMA-b-PMAA, SIONPs were prepared by coprecipitation of Fe(II) and Fe(III), yielding $\mathrm{Fe}_{3} \mathrm{O}_{4}$ SIONPs stabilized with POEOMA-b-PMAA block copolymers (Figure 4). Their diameters were 
tuned in the range of $10-25 \mathrm{~nm}$ by varying the initial copolymer concentration. The resulting polymerstabilized SIONPs with long-term colloidal stability could be useful as MRI contrast agents. ${ }^{[90]}$ The combination of ATRP of solketal acrylate (SA), functionalization with folate, and hydrolysis of PSA allowed for the preparation of well-controlled folate-functionalized poly(glycol monoacrylate) (FolatePGA). Hydroxyl groups enabled PGA to be absorbed on SIONPs, yielding folate-conjugated PGAcoated SIONPs. ${ }^{[91]}$

Reversible addition-fragmentation chain transfer (RAFT) polymerization has also been utilized. Welldefined poly(acrylic acid) (PAA) homopolymer was prepared in dimethylformamide (DMF). A subsequent RAFT polymerization yielded well-defined triblock copolymer consisting of PAA, poly(Nisopropylacrylamide) (PNIPAM), and POEOMA blocks. The resulting PAA-b-PNIPAM-b-POEOMA block copolymer was post-added to $\mathrm{Fe}_{3} \mathrm{O}_{4}$ NPs. The resulting $\mathrm{Fe}_{3} \mathrm{O}_{4}$ NPs stabilized with triblock copolymers exhibited volume change in response to external stimuli such as temperature and $\mathrm{pH}$. For example, the diameter of $\mathrm{Fe}_{3} \mathrm{O}_{4}$ NPs stabilized with $\mathrm{PAA}_{41}$-b-PNIPAM ${ }_{150}$-b-POEOMA 90 triblock copolymer decreased from 70 to $45 \mathrm{~nm}$ in response to temperature change from 39 to $25^{\circ} \mathrm{C}$. This quality is highly applicable towards hypothermia. ${ }^{[92]}$ Well-controlled poly(2-acetoacetoxyethyl methacrylate) (PAAEM-b-POEOMA) was prepared by the RAFT polymerization. Coprecipitation of $\mathrm{Fe}(\mathrm{II})$ and $\mathrm{Fe}(\mathrm{III})$ in the presence of PAAEM-b-POEOMA block copolymer yielded SIONPs stabilized with diblock copolymer, in which pendent acetoacetoxy groups anchored to the surfaces of SIONPs. ${ }^{\text {[93] }}$

In addition to CRP, other polymerization methods have been explored for preparation of wellcontrolled block polymers that can stabilize SIONPs. These polymers consist of pendent $\mathrm{COOH}$ or epoxy groups as anchoring blocks, yielding polymer-encapsulated SIONP dispersions. Examples include anionic polymerization and sequential photo-crosslinking for poly(isoprene)-b-poly(2cinnamoylethyl methacrylate)-b-PAA, ${ }^{[94]}$ polycondensation for PEO-b-poly(COOH-containing urethane)-b-PEO, ${ }^{[95]}$ ring-opening polymerization and subsequent hydrolysis for PEO-b-poly(aspartic 
acid), ${ }^{[96]}$ and ring-opening metathesis polymerization (ROMP) for diblock copolymer of bicycle[2,2,1]hept-5-ene-2-carboxylic acid oxiranylmethyl ester. ${ }^{[97]}$

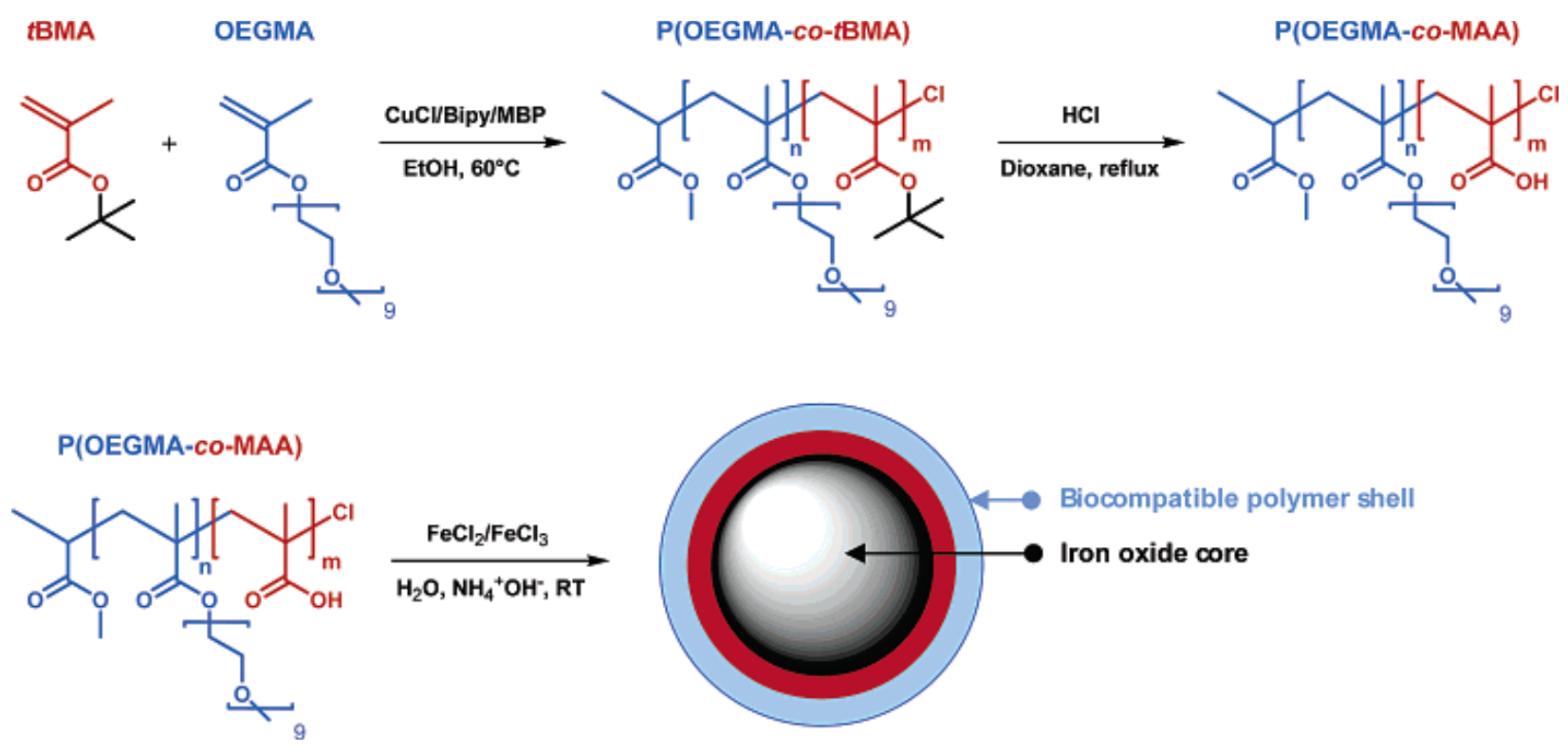

Figure 4. Schematic illustration for preparation of well-controlled POEOMA-b-PMAA copolymer and polymer-coated SIONPs. Reprinted with permission from ref ${ }^{[90]}$. Copyright 2006 American Chemical Society.

The addition of second layer approach involves the use of amphiphilic block copolymers consisting of a hydrophobic portion that intercalates the hydrophobic stabilizing ligands such as oleic acid on magnetic NPs and a hydrophilic portion that ensures water solubility of magnetic NPs. As illustrated in Figure 5, oleic acid-stabilized $\mathrm{Fe}_{3} \mathrm{O}_{4} \mathrm{NPs}$ was first prepared by thermal decomposition of iron oleate $\left(\mathrm{Fe}(\text { oleate })_{3}\right)$ in dioctyl ether. The resulting organic solution was added into an aqueous solution of Pluronic F127, an amphiphilic PEO-b-poly(propylene oxide) (PPO)-b-PEO block copolymer. The obtained oil-in-water microemulsion was dried, yielding a fine powder of $\mathrm{F} 127$-stabilized $\mathrm{Fe}_{3} \mathrm{O}_{4} \mathrm{NPs}$. They were then redispersed in water, resulting in the formation of water-soluble magnetic NPs. ${ }^{[98]}$ Amine-end-functionalized PNIPAM (PNIPAM- $\mathrm{NH}_{2}$ ) was prepared by free radical polymerization in DMF, and then reacted with poly(maleic anhydride-alt-octadecene). Magnetic NPs stabilized with the 
resulting PNIPAM-based amphiphilic block copolymer exhibited volume change in response to temperature change. ${ }^{[99]}$

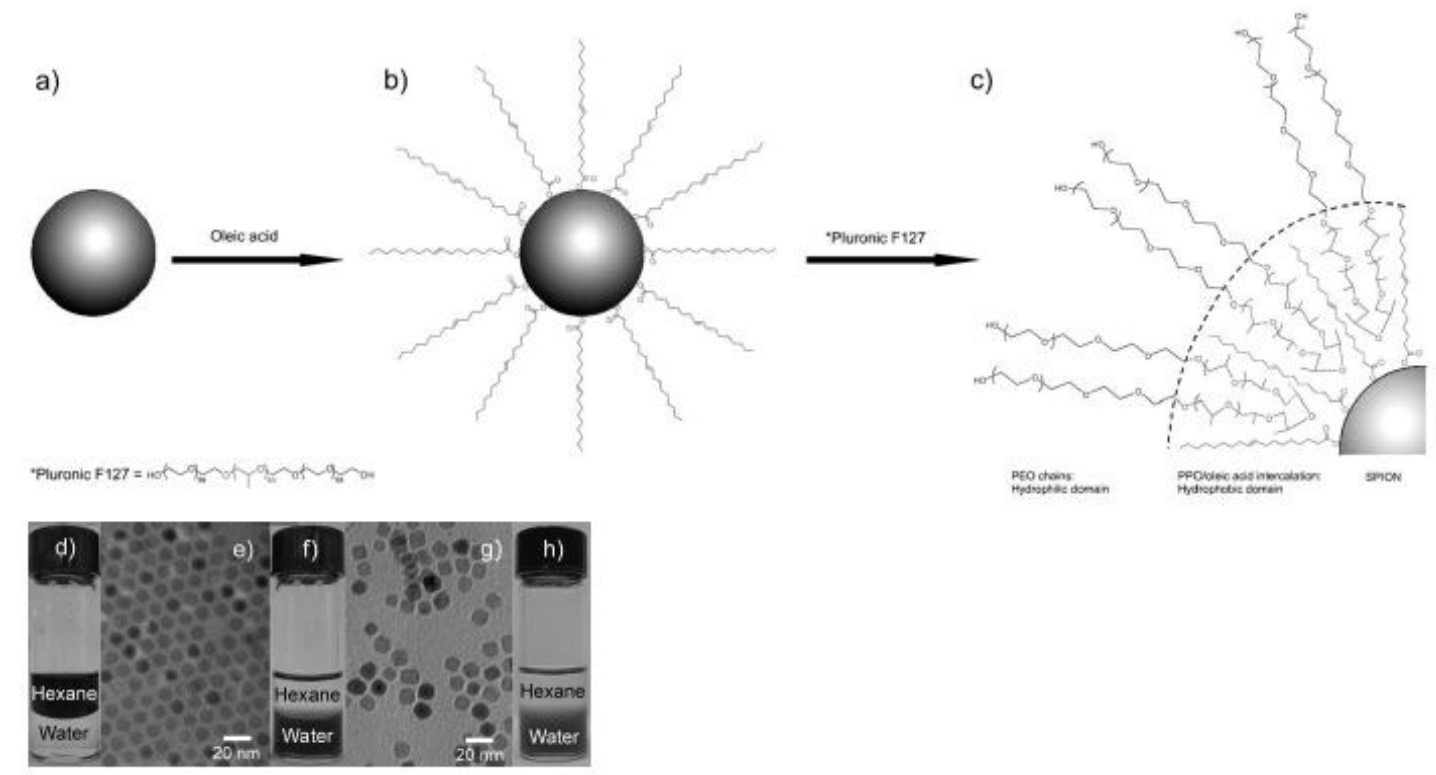

Figure 5. Schematic illustration for preparation of water-soluble magnetic NPs by addition of second layer approach, in which F127 PEO-b-PPO-PEO amphiphilic block copolymer is added into to oleic acid-stabilized magnetic NPs (a-c). Digital pictures show the distribution of magnetic NPs before $(\mathrm{d})$ and after $(\mathrm{f})$ phase transfer and F127-stabilzied magnetic NPs in water showing colloidal stability after four months (h). TEM images of magnetic NPs in hexane (e) and water (g). Reprinted with permission from ref ${ }^{[98]}$. Copyright 2007 Wiley InterScience.

For the functional silica coating approach, copolymers bearing trimethoxysilyl groups capable of crosslinking reactions on SIONPs have been prepared. Examples include copolymers consisting of poly(3-trimethoxysilyl)propyl methacrylate (PEPMA) and poly(N-acryloxysuccinimide) (PNAS). ${ }^{[100]}$ PNAS block was further functionalized with Cy5.5 fluorescent dye for in vivo tumor detection by dual magnetic resonance and fluorescence imaging. ${ }^{[101]}$ Ionic interaction between negatively charged SIONPs and positively charged poly(L-lysine) has been utilized for stem cell labeling. ${ }^{[102]}$ In addition, dopamine-conjugated hyaluronic acid (HA) ${ }^{[103]}$ polypeptide with a sequence of GGGGYSAYPDSVPMMSK (a targeting ligand for ovarian cancer cells), ${ }^{[20]}$ virus, ${ }^{[104]}$ Dex,${ }^{[105]}$ Dex- 
derivative modified with TAT-derived peptide, ${ }^{[106]}$ dopamine-plus-human serum albumin, ${ }^{[107]}$ and dendrimer $^{[108]}$ has been utilized to modify SIONPs for MR cancer imaging.

\section{Surface-initiated controlled polymerization: "grafting from" method}

The general approach for "grafting from" method involves the utilization of surface-initiated CRP methods. ATRP method has been extensively utilized for modification of single SIONP with wellcontrolled polymers because of facile functionalization of SIONPs with ATRP initiating species (halides) for surface-initiated controlled polymerization. Two routes for immobilization of halideinitiating species have been proposed. The first route involves physical absorption of acidfunctionalized halides on SIONPs. They include 3-chloropropionic acid ${ }^{[109,110]}$ 2-bormo-2-methyl propionic acid, ${ }^{[11]}$ and 10-carboxydecanyl-2-bromo-2-methyl-thiopropanoate, ${ }^{[12]}$ which initiate the ATRP of styrene (St) and OEOMA in bulk or in organic solvents, yielding single SIONP coated with hydrophobic PSt or water-soluble POEOMA. In addition, SIONPs were functionalized with 2-bormo-2methyl propionic acid, which initiated ATRP of 2-methoxyethyl methacrylate (MEMA). The resultant PMEMA-coated SIONPs exhibited quick temperature responsiveness at upper critical solution temperature (UCST) in MeOH. As seen in Figure 6, PMEMA-coated magnetic NPs were precipitated at below UCST; however at above UCST, they were redispersed to form a stable dispersion that shows collective response to a permanent magnet. ${ }^{[113]}$ The other route for the functionalization of SIONPs with ATRP initiating halides involves the covalent attachment via silanization. Examples include the immobilization of 2-(4-chlorosulfonylphenyl)ethyltrichlorosilane for poly(methyl methacrylate), ${ }^{[114]}$ [11-(2-bromo-2-methyl)-propionyloxy]undecyltrichlorosilane for PSt, ${ }^{[115]}$ and [4(chloromethyl)phenyl]trichlorosilane for POEOMA. ${ }^{[116]}$

The RAFT polymerization method has also been explored to modify single SIONP with wellcontrolled polymers. An example includes the treatment of SIONPs with ozone to create hydrogen 
peroxides, free radical initiating species. RAFT polymerization of St or acrylic acid (AA) in the presence of 1-phenylethyl dithiobenzoate (PDB) in DMF was then carried out, yielding PSt or PAAgrafted magnetic NPs. They were characterized with X-ray photoelectron spectroscopy (XPS), FT-IR spectroscopy, and gel permeation chromatography (GPC). ${ }^{[117]}$ Another example include the ligand exchange oleic acid on $\mathrm{Fe}_{3} \mathrm{O}_{4}$ with $\mathrm{S}$-1-dodecyl-S'-( $\alpha$, $\alpha^{\prime}$-dimethyl- $\alpha$ "-acetic acid)trithiocarbonate (DDMAT), a RAFT agent, followed by RAFT polymerization of NIPAM. The resulting PNIPAMcoated SIONPs exhibited thermoresponsiveness. ${ }^{[118]}$

In addition to the "grafting from" method, the "grafting onto" method has also been utilized for the preparation of SIONPs coated with single layer of polymers. For the method, polymers are designed to be monodentates that possess a terminal anchoring group at the end of polymer, or made of hyperbranched architectures with functionalities introduced in the focal points. Examples include thiolterminated $\mathrm{PSt}^{[119]}$ and phosphonate-functionalized PEO. ${ }^{[120]}$

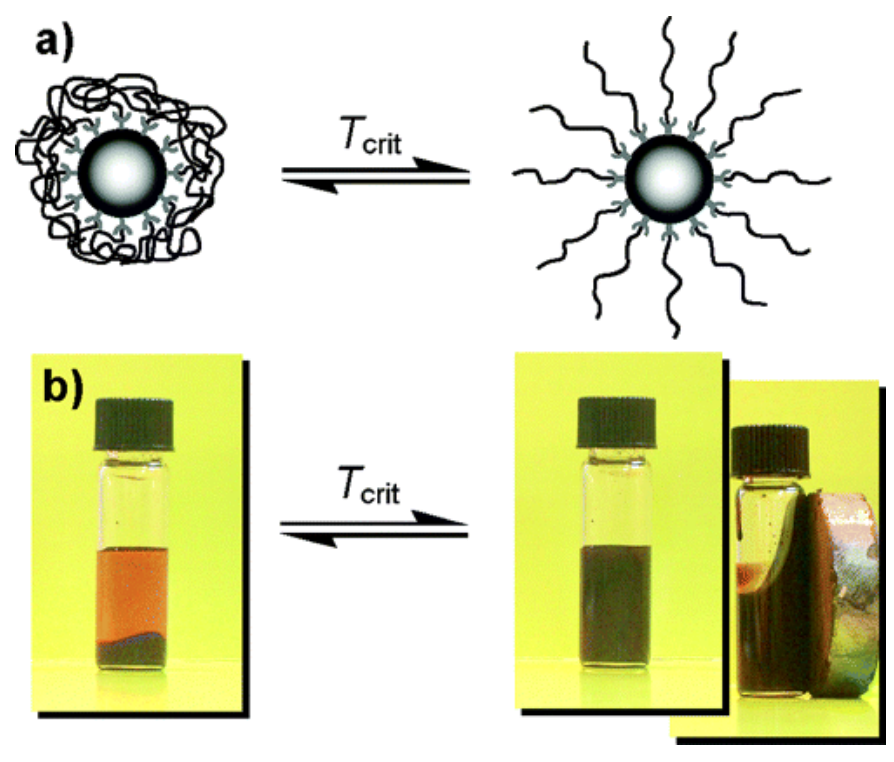

Figure 6. Schematic illustration (a) and photographs (b) of thermoresponsive PMEMA-coated magnetic NPs. The particles precipitate at below UCST; at above UCST, they are redispersed that reacts collectively under the influence of a permanent magnet. Reprinted with permission from ref ${ }^{[13]}$. Copyright 2006 American Chemical Society. 


\section{Inorganic silica/polymer hybridization}

SIONPs prepared by either coprecipitation or thermal decomposition are encapsulated with a silica shell through a sol-gel process of tetraethyl orthosilicate (TEOS). The resulting silica-coated SIONPs were further functionalized and encapsulated with polymers, yielding multifunctional hybrid nanomaterials. The preparation of hairy hybrid nanomaterials consisting of magnetic core, fluorescent silica shell, and functional polymer brushes is illustrated in Figure 7. This approach began with the solgel reaction in the presence of $\mathrm{Fe}_{3} \mathrm{O}_{4}$ NPs including fluorescein isothiocyanate (FITC), a fluorescent dye, producing $\mathrm{Fe}_{3} \mathrm{O}_{4}$ core-fluorescent silica shell, in which FITC is covalently incorporated. The resultant silica shell was functionalized with methacrylates by reacting with 3methacryloxypropyltrimethosysilane (MPS) and then chlorines via radical crosslinking polymerization of ethylene glycol dimethacrylate (EGDMA) and vinyl benzyl chloride. The chlorine groups served as initiators for ATRP of OEOMA, yielding hybrid nanomaterials with biocompatible POEOMA brushes. ${ }^{[121]}$ Silica-coated $\mathrm{Fe}_{3} \mathrm{O}_{4}$ SIONPs prepared by the sol-gel reaction of TEOS were encapsulated with crosslinked polyphosphazene, yielding pomegranate-like core/shell structured nanomaterials. ${ }^{[122]}$ In addition, sol-gel reaction of TEOS in the presence of $\mathrm{Fe}_{3} \mathrm{O}_{4} \mathrm{NPs}$ and cetyltrimethylamonium bromide (CTAB), functionalization with MPS, and then removal of CTAB yielded methacrylate-functionalized, $\mathrm{Fe}_{3} \mathrm{O}_{4}$-embedded silica nanomaterials with channels. They were coated with thermoresponsive polymeric shells consisting of PNIPAM copolymers for drug delivery applications. ${ }^{[123]}$

In another approach, amino-functionalized silica-coated $\mathrm{Fe}_{3} \mathrm{O}_{4}$ SIONPs were prepared by the reaction of silica-coated $\mathrm{Fe}_{3} \mathrm{O}_{4}$ with 3-aminoporpyltirethyoxysilane (APS). They were mixed with acidfunctionalized core/shell microgels consisting of P(St-co-NIPAM) core and crosslinked PNIPAM shell. The removal of core P(St-co-NIPAM) by dissolving in tetrahydrofuran (THF) yielded crosslinked PNIPAM capsules surface-anchored with silica-coated $\mathrm{Fe}_{3} \mathrm{O}_{4}$ SIONPs (Figure 8). ${ }^{[124]}$ 


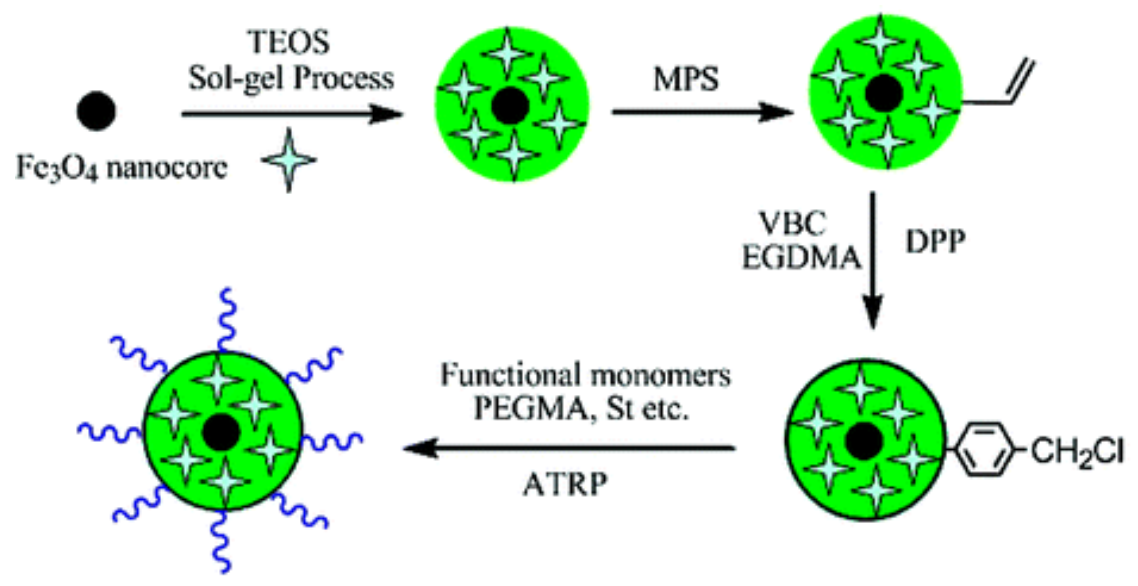

TEOS: $\mathrm{Si}-(\mathrm{OEt})_{4}$

MPS: $\left(\mathrm{CH}_{3} \mathrm{O}\right)_{3} \mathrm{Si}\left(\mathrm{CH}_{2}\right)_{3} \mathrm{OCOC}\left(\mathrm{CH}_{3}\right)=\mathrm{CH}_{2}$

VBC: $\mathrm{CH}_{2}=\mathrm{CH}-1-\mathrm{CH}_{2} \mathrm{Cl}$ EGDMA: $\mathrm{CH}_{2}=\mathrm{C}\left(\mathrm{CH}_{3}\right) \mathrm{COO}-\mathrm{CH}_{2} \mathrm{CH}_{2}-\mathrm{OCOC}\left(\mathrm{CH}_{3}\right)=\mathrm{CH}_{2}$

PEGMA: $\mathrm{CH}_{2}=\mathrm{C}\left(\mathrm{CH}_{3}\right) \mathrm{CO}\left(\mathrm{OCH}_{2} \mathrm{CH}_{2}\right)_{n}-\mathrm{OH} \quad \mathrm{St}: \mathrm{CH}_{2}=\mathrm{CH}-\mathrm{O}$

ATRP: Atom tranfer radical polymerization

DPP: Distillation precipitation polymerization

$\hat{\gamma}$ : Fluorescein isothiocyanate (FITC) fluorescent dye

Figure 7. Synthesis of hairy hybrid nanomaterials with a magnetic core, fluorescent silica shell, and functional polymer brushes. Reprinted with permission from ref ${ }^{[121]}$. Copyright 2009 American Chemical Society.
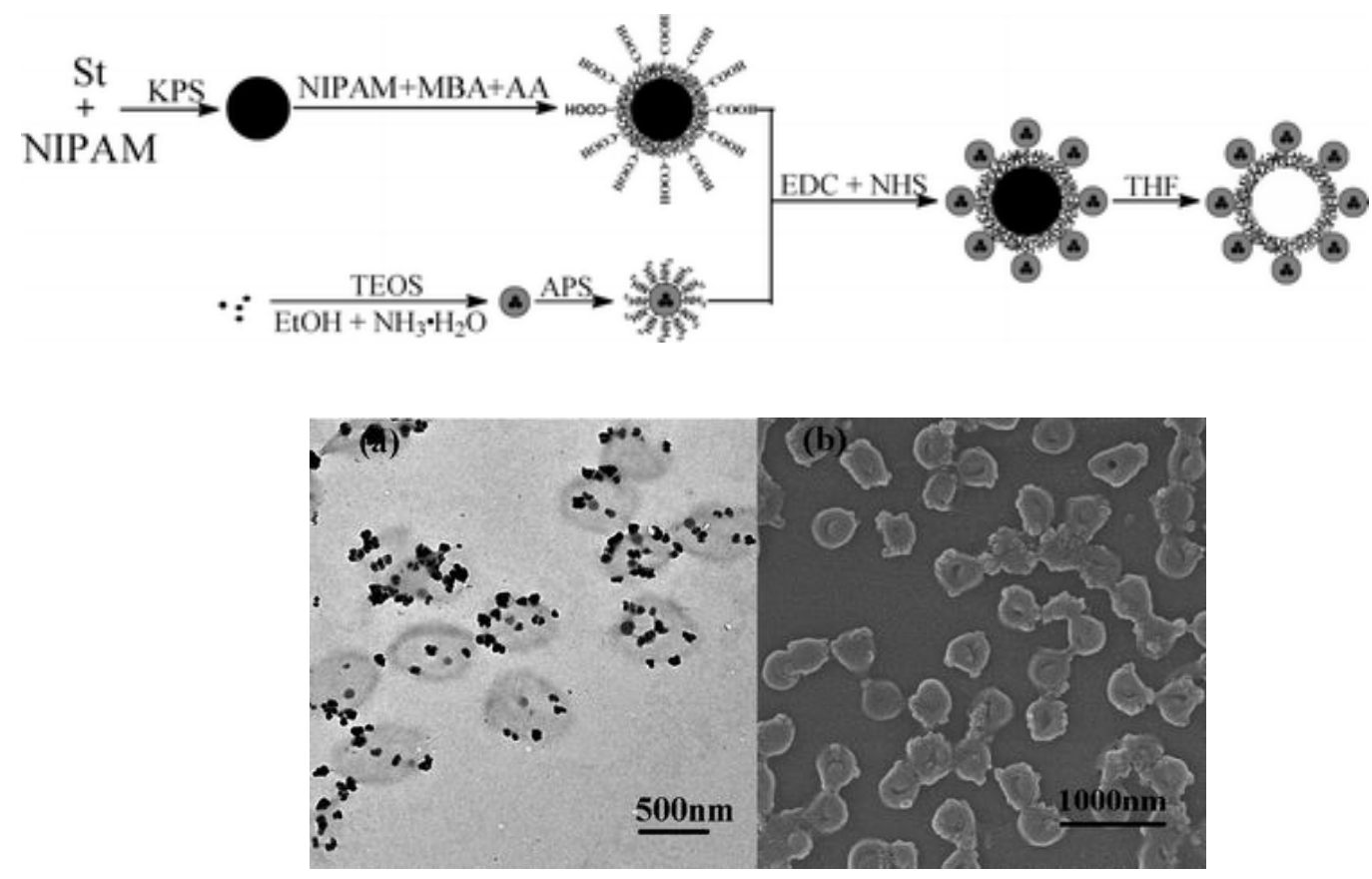

Figure 8. Preparation (upper) and TEM (a) and SEM (b) images of crosslinked PNIPAM capsules surface-anchored with silica-coated $\mathrm{Fe}_{3} \mathrm{O}_{4} \mathrm{NPs}$ (lower). Reprinted with permission from ref ${ }^{[124]}$. Copyright 2009 American Chemical Society. 


\section{Self-assembly and self-association}

Self-assembly method involves the design and preparation of amphiphilic block copolymers that enable self-assembly in water, forming stable core/shell micellar NPs wherein the hydrophobic core serves as a carrier for SIONPs and anticancer drugs and the hydrophilic shell allows particle stabilization in aqueous solution. Hydrophobic SIONPs stabilized with oleic acid are physically embedded in micellar particles through self-assembly. Further targeting ligands are attached to the surface of self-assembled NPs embedded with SIONPs and anticancer drugs for multifunctional nanomedicine platform. Polyester-based amphiphilic block copolymers were generally prepared by ring opening polymerization (ROP) of D,L-lactide (LA) and $\varepsilon$-caprolactone (CL). Well-defined COOHterminated PEO-b-PLA amphiphilic block copolymer self-assembled in the presence of hydrophobic SIONPs and doxorubicin (Dox). The resulting core/shell NPs embedded with SIONPs and Dox were then functionalized with therapeutic antibodies (targeting species to tumor) for an ultrasensitive MRI probe. It was reported that $\mathrm{Mn}$-doped $\mathrm{Fe}_{3} \mathrm{O}_{4}\left(\mathrm{MnFe}_{3} \mathrm{O}_{4}\right)$ is more efficient of $\mathrm{T}_{2}$ relaxivity for MRI than $\mathrm{Fe}_{3} \mathrm{O}_{4}$ NPs. ${ }^{[125]}$ Well-controlled PLA-b-POEOMA amphiphilic block copolymer was prepared by a combination of ROP and ATRP from 2-hydroxyethyl-2'-methyl-2'-bromo-propionate, a double-headed initiator. The copolymer self-assembled in the presence of hydrophobic $\mathrm{Fe}_{3} \mathrm{O}_{4} \mathrm{NPs}$, and further functionalized with folates for cancer cell targeting (Figure 9). ${ }^{[126]}$ Maleimide-terminated PEO-b- PLA and methoxy-terminated PEO-b-PLA self-assembled in the presence of Dox and SIONPs through mixed micellization. The resulting maleimide-functionalized polymeric NPs reacted with RGD tripeptide that can target integrin $\alpha_{\vee} \beta_{3}$ on tumor endothelial cells. ${ }^{[127]}$ In addition, the preparation and self-assembly of folate-encoded PEO-b-PCL amphiphilic block polymers, ${ }^{[128]}$ PEO-modified PSt,${ }^{[129,130]}$ and PEGphospholipid $^{[131]}$ amphiphilic block copolymers have been explored. 


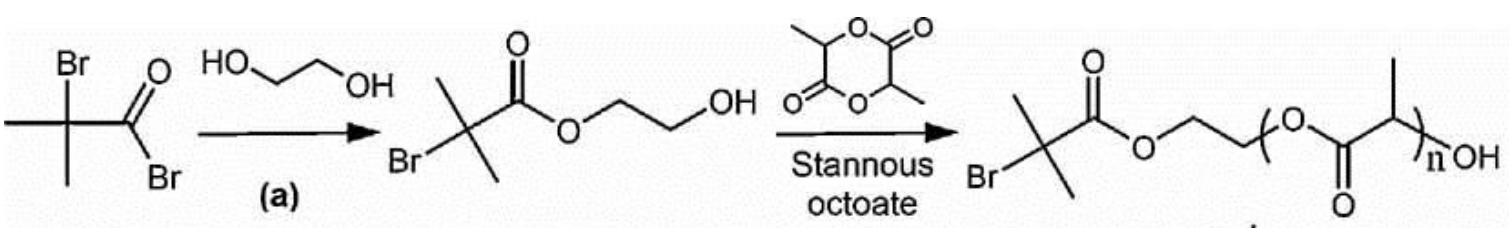

(b)

PEGMA $\mid \begin{array}{ll}\text { Bpy, } & \\ \mathrm{CuBr} & \text { (c) }\end{array}$

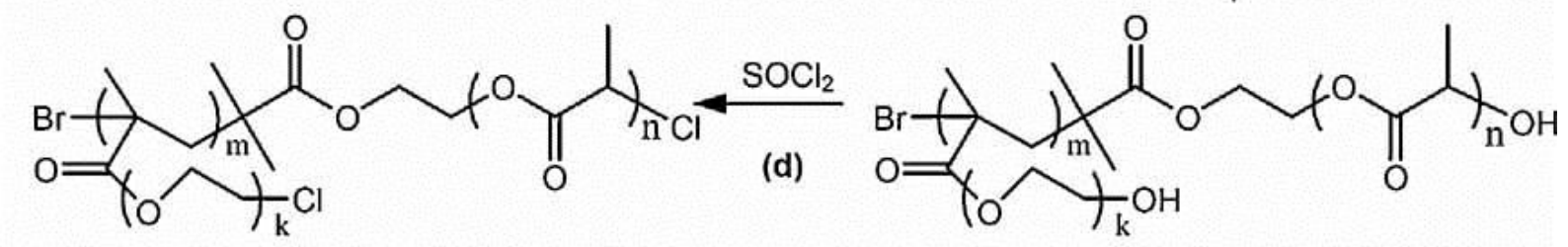

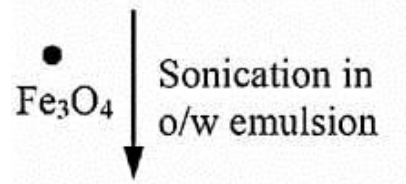

(e)

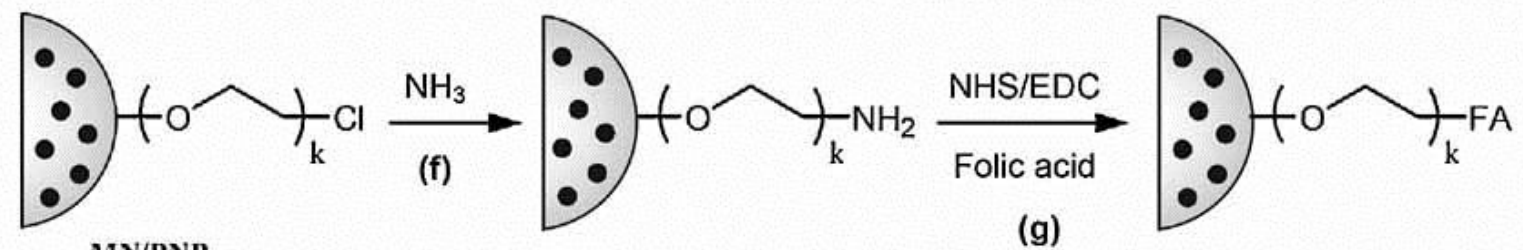

MN/PNP

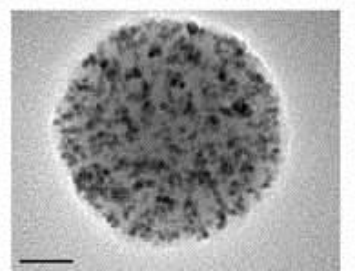

Figure 9. Schematic illustration to prepare folate-functionalized micellar NPs embedded with SIONPs from well-controlled PLA-b-POEOMA amphiphilic block copolymer by a combination of ROP and ATRP. Reprinted with permission from ref ${ }^{[126]}$. Copyright 2009 Wiley InterScience.

Self-association is generally achieved through ionic interaction between SIONPs and anionic-or cationic polymers. Examples include ionic interactions of positively-charged SIONPs/negativelycharged PAMAM dendrimers, ${ }^{[132]}$ negatively-charged SIONPs (with PAA)/poly(trimethylammonium ethyl acrylate methylsulfate)-b-PAAm, ${ }^{[133]}$ and vesicle aggregates crosslinked by positively-charged SIONPs. ${ }^{[134]}$ Interestingly, positively-charged poly(L-lysine) (PLK) mixed with negatively-charged SIONPs modified with citrate ions resulted in self-complex coacervates, which were then post- 
crosslinked with glutaraldehyde (GA) crosslinkers to obtain PLK-MIONP hybrid microspheres. ${ }^{[135,136]}$ In addition, self-complexation through hydrophobic interactions has been explored. An example includes inclusion interactions of $\beta$-cyclodextrins with oleic acid-stabilized SIONPs. ${ }^{[137]}$

Emulsification/solvent evaporation technique has also been explored for the preparation of polymeric NPs based on PLA homopolymers embedded with hydrophobic SIONPs. For the approach, hydrophobic PLA dissolved in volatile organic solvents were mixed with aqueous surfactant solution, forming oil-in-water emulsion. Solvents were then evaporated, yielding stable PLA-based NPs embedded with SIONPs in water with an aid of surfactants. ${ }^{[138-140]}$

\section{Heterogeneous polymerization}

Various heterogeneous polymerization reactions of hydrophilic or water-soluble monomers have been explored to prepare well-defined SIONP-embedded magnetic spheres as well as crosslinked microgels/nanogels and hydrogels for biomedical applications. These reactions include inverse (mini)emulsion polymerization, dispersion polymerization, and precipitation polymerization. In addition, heterogeneous polymerization of hydrophobic monomers such as styrene in the presence of hydrophobic SIONPs in conventional emulsion, miniemulsion, and suspension has produced magnetic hydrophobic particles. ${ }^{[141-149]}$ This review concentrates on the preparation of hydrophilic magnetic particles for biomedical applications.

\subsection{Inverse (mini)emulsion polymerization}

Inverse (mini)emulsion polymerization is a water-in-oil $(\mathrm{W} / \mathrm{O})$ polymerization process that contains aqueous droplets consisting of water-soluble monomers stably dispersed with the aid of oil-soluble surfactants in a continuous organic medium. Stable dispersions are formed by mechanical stirring for inverse emulsion process and by sonification for inverse miniemulsion polymerization. When radical 
initiators are added, polymerization occurs within the aqueous droplets producing colloidal particles. ${ }^{[150]}$ An introduction of multifunctional crosslinkers allows for preparation of crosslinked microgel particles. ${ }^{[151]}$ When the size of microgels is in submicron-sized, it is defined as nanogels. Several reports have demonstrated the use of inverse (mini)emulsion polymerization for the preparation of hydrophilic or water-soluble polymeric particles ${ }^{[152-154]}$ and crosslinked microgels/nanogels. ${ }^{[155-160]}$ Recently, a unique method utilizing controlled ATRP in inverse miniemulsion has been developed for the preparation, functionalization, and application of well-defined biodegradable nanogels for targeted drug delivery. ${ }^{[161-168]}$ The details are reported elsewhere. ${ }^{[169]}$

Inverse miniemulsion polymerization has been explored to prepare well-defined hybrid magnetic polymer particles. This method requires the preparation of hydrophilic or water-soluble SIONPs. ${ }^{[170]}$ An example includes the preparation of magnetic PAAm-based microgels with a diameter of $60-160 \mathrm{~nm}$. An aqueous homogeneous solution of AAm, N,N'-methylenebisacrylamide (MBAm), and MAAstabilized magnetic fluid in a dilute aqueous ammonia was mixed with an organic solution of Span 80 (sorbitol monooleate) in cyclohexane under stirring. The resulting bi-phase was sonicated and polymerized upon the addition of free-radical initiator, producing stable miniemulsion of magnetic PAAm-microgels with a diameter of $100 \mathrm{~nm}$. The magnetite content in the particles was determined to be $13 \mathrm{wt} \%$, which was consistent with the concentration of magnetite NPs in the feed. ${ }^{[171]}$

Inverse emulsion/microemulsion polymerization has also produced well-defined magnetic polymer particles. Aqueous droplets of MAA, 2-hydroxyethyl methacrylate (2-HEMA), and SIONPs were dispersed in a solution of sodium dioctylsulfosuccinate in toluene. Copolymerization of the monomers yielded hydrophilic polymer beads physically loaded with magnetic NPs, yielding composite magnetic particles of hydrophilic polymers. However, they were relatively polydisperse and only contained 3.3 wt\% magnetic NPs. ${ }^{[172]}$ Several approaches have been proposed to increase loading level of SIONPs into polymeric particles. One approach involves stabilization of SIONPs with double-hydrophilic block 
copolymer, PEO- $b$-PMAA. A water soluble monomer mixture containing SIONPs stabilized with PEOb-PMAA, 2-HEMA, and MAA was mixed with organic solution of poly(ethene-co-butene)-b-PEO in decane. The addition of an organic initiator yielded magnetic microspheres with a diameter of 50-250 $\mathrm{nm}$ and a loading level of SIONPs up to $18 \mathrm{wt} \%{ }^{[173]}$ Another approach involves albeit the size uniformity. The magnetic loading further increased to $23 \%$. Submicrometer-sized magnetic hydrophilic polymer particles were prepared by inverse microemulsion polymerization of AAm, MBAm, and suspension of trisodiumcitrate-stabilized SIONPs dispersed in a solution of sodium bis(2-ethylhexyl) sulfosuccinate in toluene. The resulting magnetic PAAm microgels had their particle size ranging from 80 to $180 \mathrm{~nm}$ in diameter, which can be controlled by the concentration of MBAm crosslinker and surfactant/water ratio. The magnetite content in polymer particles was determined to be $5-23 \mathrm{wt} \%{ }^{[174]}$

\subsection{Dispersion polymerization}

Dispersion polymerization is advantageous because micrometer-size polymer microspheres with a narrow size distribution can be obtained in a single step. Of utmost importance for the technique is the appropriate selection of the reaction medium, in which monomers are soluble, while the resulting polymer and magnetic material are insoluble. By heating the polymerization mixture, initiator is decomposed to form oligomer radicals. The oligomeric chains do not remain dissolved in the medium, but precipitate when reaching the critical chain length. The chains associate forming nuclei, which aggregate and at the same time adsorb the stabilizer forming primary mature particles containing magnetic cores. Under conditions where no new nuclei are formed, the primary particles grow and reach the uniform size. ${ }^{[175-177]}$

Dispersion polymerization was conducted with 2-HEMA in the presence of iron oxide needles or cubes (ca. 100 500 nm) in a mixture of toluene/2-methylpropan-1-ol, yielding magnetic microspheres with a diameter of 1-2 $\mu \mathrm{m}$ stabilized with acetate butyrate cellulose. Ethylene glycol dimethacrylate 
(EGDMA) was added to prepare crosslinked microspheres. ${ }^{[178]}$ Similar process was applied to prepare magnetic microspheres of PGMA ${ }^{[179-181]}$ and PAAm ${ }^{[182]}$ stabilized with poly(vinyl pyrrolidone) (PVP). In addition, magnetic microspheres of $\mathrm{P}(\mathrm{St}-\mathrm{co}-\mathrm{GMA})$ with $72 \mathrm{wt} \%$ of magnetic content measured by thermal analysis methods were prepared. ${ }^{[183]}$

Thermoresponsive polymeric microgels loaded with SIONPs and anti-cancer drugs are of interest for multi-functional cancer therapies. This is because such nanoparticles can be used for magnetic drug targeting followed by simultaneous hyperthermia and drug release. $\gamma-\mathrm{Fe}_{2} \mathrm{O}_{3}$ SIONPs with diameter of 14 , 19, and $43 \mathrm{~nm}$ were synthesized by high temperature decomposition. Composite magnetic nanoparticles of PNIPAM were prepared by aqueous dispersion polymerization of NIPAM in the presence of SIONPs. As seen in Figure 10, thermo-responsiveness of PNIPAM exhibited facile loading and release of drugs at temperatures below and above the lower critical solution temperature $(34 \circ \mathrm{C})$. The particles showed Fickian diffusion release kinetics; the maximum Dox release at $42 \circ \mathrm{C}$ after $101 \mathrm{~h}$ was $41 \%$. In vitro simultaneous hyperthermia and drug release of therapeutically relevant quantities of Dox was achieved. For example, $14.7 \%$ of loaded Dox was released in $47 \mathrm{~min}$ at hyperthermia temperatures. ${ }^{[184]}$ 
(a)

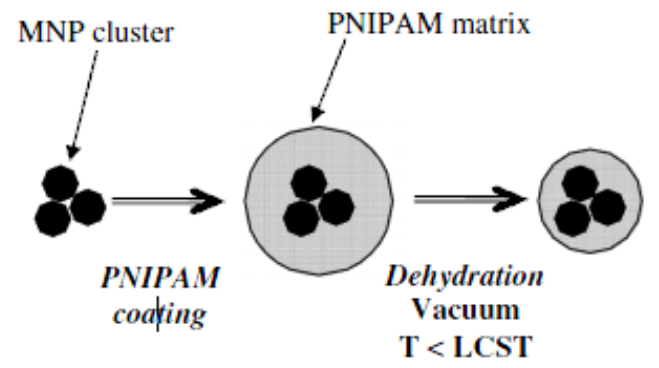

(b)

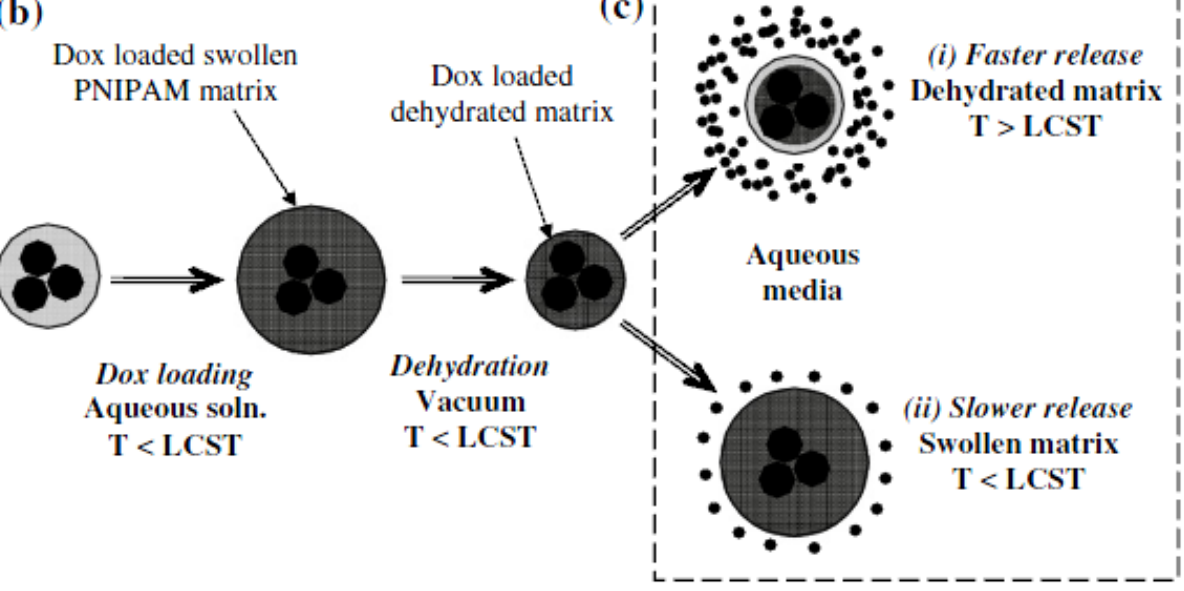

Figure 10. Schematic overview of composite magnetic microsphere preparation(a), drug loading(b), and drug release processes(c). Reprinted with permission from ref ${ }^{[184]}$. Copyright 2009 IOP.

\subsection{Other heterogeneous polymerization methods}

Precipitation polymerization of hydrophilic or water-soluble monomers in the presence of crosslinkers in water produces microgel particles. Typical examples are thermoresponsive PNIPAM-based microgels. Carboxylic acid-functionalized P(NIPAM-HEA-AA) microgels were prepared, and used as reactors for in-situ formation of inorganic NPs including $\mathrm{Fe}_{3} \mathrm{O}_{4} \mathrm{NPs}^{\left[{ }^{[185]}\right.}$ After being immersed in aqueous solution of $\mathrm{Fe}(\mathrm{II})$ and $\mathrm{Fe}(\mathrm{III})$, microgels of poly(acetoacetoxyethyl methacrylate-co-N-vinylcarprolactam) (P(AAEM-VCL)) became temperature-sensitive hybrid microgels with magnetic properties. ${ }^{[186]}$ Thermally-responsive magnetic microgels of poly(di(ethylene glycol) methyl ether methacrylate) $\left(\mathrm{PMe}(\mathrm{EO})_{2} \mathrm{MA}\right)$ crosslinked with disulfides were prepared by ATRP under emulsion conditions. They 
were then mixed with oleic acid-stabilized SIONPs, magnetic microgels, followed by physical loading of Rhodamine B. Upon addition of reducing agents, the microgels degraded to release the hydrophilic drugs. ${ }^{[187]}$ In addition, the preparation of magnetic polyvinylamine NPs by in situ precipitation polymerization was reported. ${ }^{[188]}$

\section{Bulk physical and chemical crosslinking - magnetic hydrogel preparation}

Magnetic hydrogels containing magnetic NPs, called ferrogels, have been prepared by both physical and chemical crosslinking reactions in the presence SIONPs. For physical crosslinking, poly(vinyl alcohol) (PVOH) with degree of hydrolyzation of $>97 \%$ was mixed with dimethylsulfoxide (DMSO) at $80{ }^{\circ} \mathrm{C}$ under stirring and then SIONPs under ultrasonification. The resulting mixture was subjected to five freeze-thaw cycles at $-20{ }^{\circ} \mathrm{C}$ for $16 \mathrm{~h}$ and $25^{\circ} \mathrm{C}$ for $5 \mathrm{~h}$. The resulting physically-crosslinked PVOH-based hydrogels exhibited controlled release of drugs upon external application of magnetic field due to a precise control of opening and closure of pore configuration. ${ }^{[189,190]}$ Collagen molecules selfassembled into higher-order structure when $\mathrm{pH}$ of collagen solution increased to 7.4 at $37{ }^{\circ} \mathrm{C}$. An addition of SIONP solution at $\mathrm{pH}=4$ resulted in the formation of physically-crosslinked collagen gels. The gels were further stabilized by a carbodiimide coupling reaction in the presence of N-(3dimethylaminopropyl)-N'-ethyl-carbodiimide (EDAC). Rhodamine-labeled Dex was incorporated into magnetic collagen gels for controlled release of Dex, a model drug, in external magnetic field. ${ }^{[191]}$

For chemical crosslinking, free-radical crosslinking polymerization in water has been utilized. Thermosensitive hydrogels of alginate-PNIPAM semi-interpenetrating networks (IPN) embedded with SIONPs were prepared by simultaneous free-radical crosslinking polymerization of NIPAM with MBAm and physical crosslinking of Alg with $\mathrm{Ca}^{2+}$ ions. The alginate-PNIPAM IPN gels had larger pores than PNIPAM gels, exhibiting fast response to temperature, and thus leading to high rate of 
swelling/deswelling. The resulting cylindrically shaped gels of $20 \mathrm{~mm}$ were immersed in an aqueous basic solution of $\mathrm{Fe}(\mathrm{II})$ and $\mathrm{Fe}(\mathrm{III})$, yielding magnetic hydrogels for hyperthermia applications. ${ }^{[192,193]}$

\section{Bio-applications of SIONP-polymer hybrids}

This section discusses bio-related applications of hybrid magnetic polymer particles embedded with SIONPs. They include MR imaging (or dual imaging with optical imaging based on fluorescence), targeted drug delivery, hypothermia, protein immobilization, and biosensors.

\subsection{MR imaging}

MR imaging is one of the most powerful non-invasive imaging methods utilized in clinical medicine, which is based on the relaxation of protons in tissues. Upon accumulation in tissues, SIONPs enhance proton relaxation of specific tissues when compared with the surrounding tissues, serving as a MR contrast agent. For in vivo MR imaging applications, SIONPs should have longer half-life time in the blood circulation for the improved efficiencies of detection, diagnosis, and therapeutic management of solid tumors. Because opsonin plasma proteins are capable for interacting with plasma cell receptors on monocytes and macrophages, opsonin-absorbed SIONPs will be quickly cleaned by circulating monocytes or fixed macrophages through phagocytosis, leading to elimination of SIONPs from blood circulation. The smaller the particle and the more neutral and hydrophilic its surface, the longer is its plasma half-life. Therefore, the surface of SIONPs has been modified with hydrophilic polymers to prevent absorption of the circulating plasma proteins.

POEOMA-coated SIONPs were incubated with RAW 264.7 microphage cells and the extent of their cellular uptake was compared with pristine SIONPs. As seen in Figure 11, iron concentration in RAW264.7 cells incubated with POEOMA-coated SIONPs is much smaller than that with pristine SIONPs, indicating the importance of surface properties of SIONPs for in vitro and in vivo MR imaging

applications. ${ }^{[16]}$ SIONPs coated with POEOMA-b-PMAA block copolymer were injected into a rat. 
Figure 12 shows the in vivo MR images of liver section of the live rat over time after injection. The liver was observed to be significantly darker after $2 \mathrm{~h}$, which is much longer than 5 min for Resovist ${ }^{\circledR}$, a larger commercial SIONP. The results suggest that ultra small SIONPs coated with POEOMA (diameter $=10 \mathrm{~nm}$ ) have a longer half-life time in the bloodstream than standard commercial contrast agent. ${ }^{[0]}$



Figure 11. Iron concentration in RAW 264.7 cells cultured in medium containing pristine (a) and POEOMA-coated SIONPs (b). Reprinted with permission from ref ${ }^{[116]}$. Copyright 2006 American Chemical Society. 


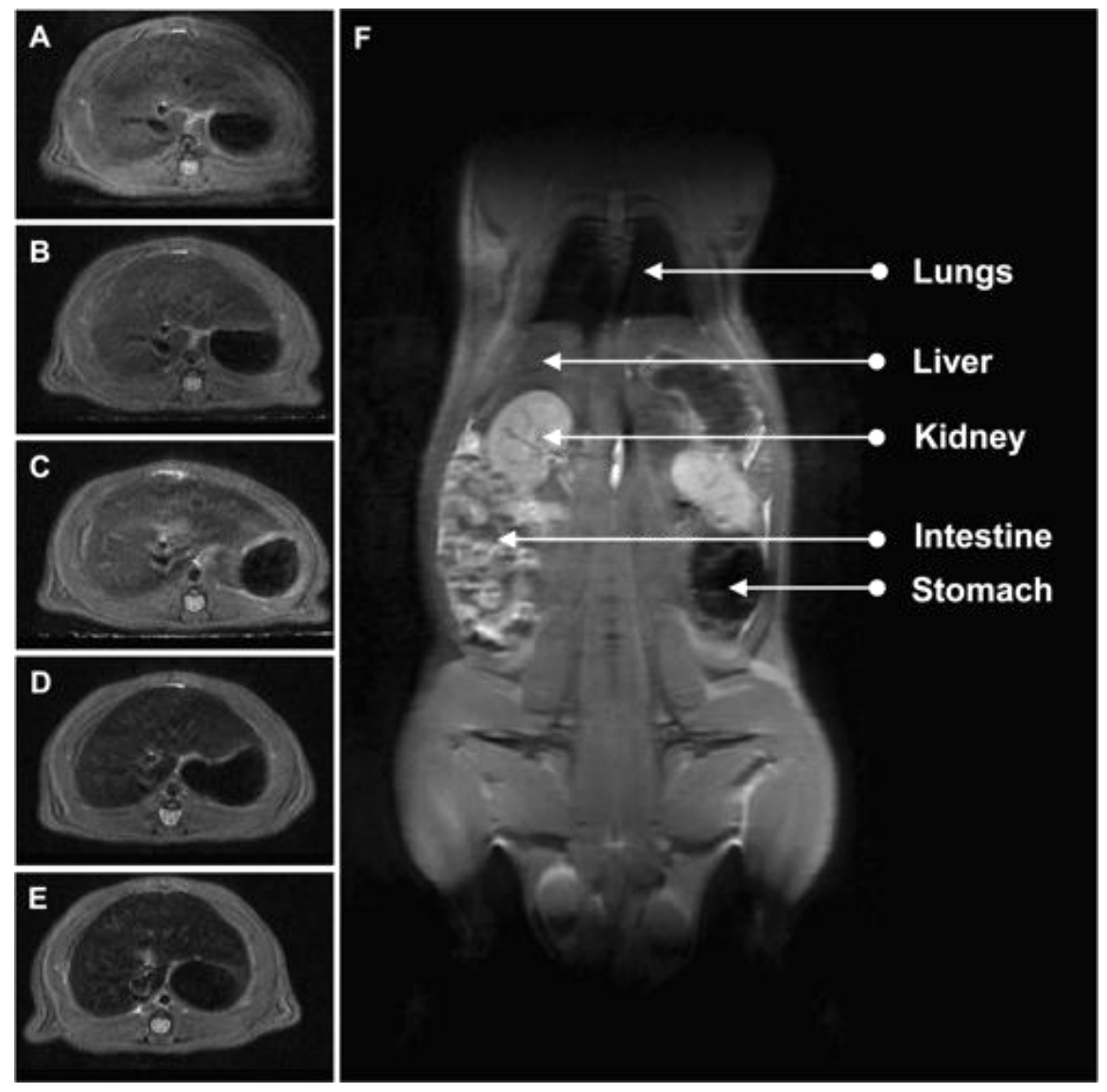

Figure 12. MR Images of a live rat after injection of $500 \mu \mathrm{L}$ of a solution containing SIONPs coated with POEOMA-b-PMAA $(\mathrm{d}=10 \mathrm{~nm})$. Images on the left show liver selections measured at 0 min (a), $15 \mathrm{~min}(\mathrm{~b}), 1 \mathrm{~h} \mathrm{(c),} 2 \mathrm{~h}(\mathrm{~d}), 6 \mathrm{~h}$ (e). Right image shows a coronal section measured after 70 min. Reprinted with permission from ref ${ }^{[90]}$. Copyright 2006 American Chemical Society.

SIONPs coated with hydrophilic polymers, typically POEOMA and poly(L-lysine) have also been utilized for in vitro and in vivo labeling cancer and stem cells. They were accumulated in tissues by enhance permeability and retention (EPR) effect for MR imaging of specific cells. ${ }^{[100,}{ }^{136]} \mathrm{Fe}_{2} \mathrm{O}_{3}$ maghemite NPs coated with poly(N,N-dimethylacrylamide) (PDMAAm) were prepared by solution radical polymerization of DMAAm in the presence of aqueous solution of maghemite NPs, which were prepared by the coprecipitation method. In vitro cellular uptake results indicate that PDMAAm-coated 
$\mathrm{Fe}_{2} \mathrm{O}_{3}$ maghemite NPs exhibited higher efficiency in labeling rat and human bone marrow mesenchymal stem cells than pristine $\mathrm{Fe}_{2} \mathrm{O}_{3}$ NPs and even Dex-modified NPs (Endorem ${ }^{\circledR}$, a commercial MRI contrast enhancement agent $)^{[194,195]}$ In addition, Cy5.5, a fluorescent-dye, was incorporated into polymer/SIONPs for dual MR and fluorescence imaging. The resulting Cy5.5-conjugated polymer/SIONPs were injected intravenously into the rat through its tail vain. They were accumulated in tumor by EPR effect, as seen in vivo MR and fluorescence images of tumor after injection (Figure 13). ${ }^{[101]}$

(a)
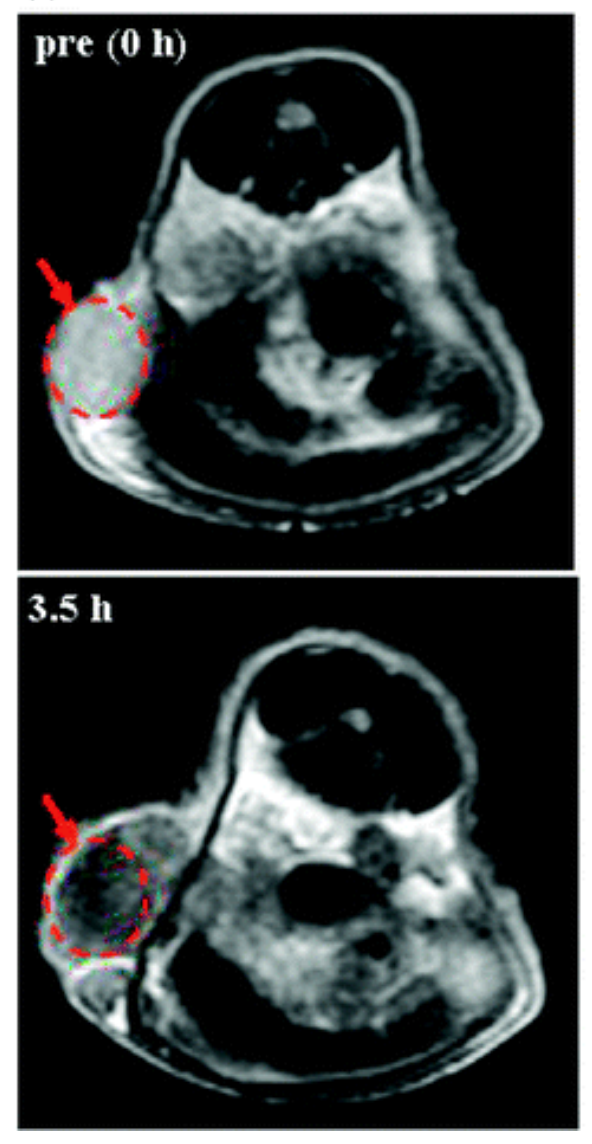

(b)
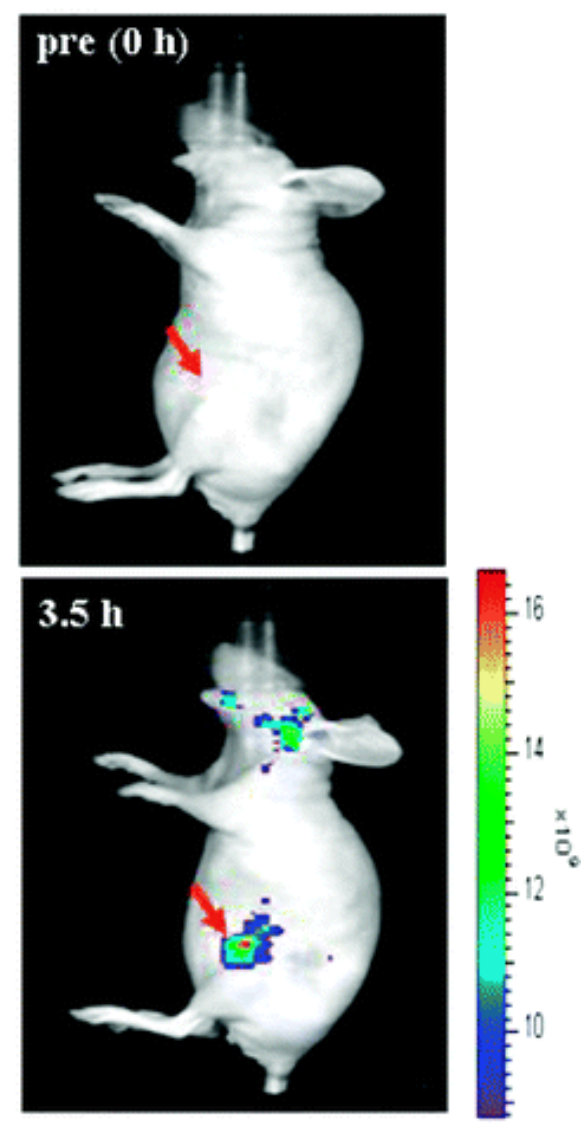

Figure 13. $\mathrm{T}_{2}$-weighted fast spin-echo images taken at 0 and $3.5 \mathrm{~h}$ post-injection of $14.7 \mathrm{mg}$ of Cy5.5-conjugated SIONPs coated with POEOMA at the level of tumor $(320 \mathrm{~mm} 3)$ on the flak above the upper left thigh of a nude mouse (a) and optical fluorescence images of the same mouse taken at 0 and $3.5 \mathrm{~h} \mathrm{(b)}$. The red arrows indicate the position of the allograft tumor. Reprinted with permission from ref ${ }^{[101]}$. Copyright 2007 American Chemical Society. 
Active targeting or specific targeting is a promising approach toward increasing the local accumulation of SIONPs in diseased tissue. ${ }^{[8,196]}$ This approach requires the design and preparation of SIONPs coated with functional polymers, which are further conjugated with targeting biomolecules to specific cells. The effective targeting biomolecules include folic acid and its analogues, peptides, proteins, and antibodies, utilizing specific interactions such as receptor-ligand or antigen-antibody interactions. Folate ${ }^{[91,126]}$ Tat peptide, ${ }^{[106]}$ and a polypeptide with a sequence of GGGGYSAYPDSVPMMSK ${ }^{[20]}$ have been conjugated to SIONPs through functional polymers for in vitro and in vivo targeting cancer cells. Dopamine-functionalized hyaluronic acid (HA) was conjugated with SIONPs in water, yielding stable HA-SPIONPs with a diameter of $15 \mathrm{~nm}$ on mica surface by atomic force microscopy (AFM). They were cultured with CD44+ cells (HCT116, human colon carcinoma cell line) and CD44- fibroblast cells (NIH3T3, mouse fibroblast). As seen in Figure 14, the MR imaging revealed that the cellular uptake of HA-SIONPs was greatly enhanced in HCT116 by specific CD44-HA receptor-ligand interactions. ${ }^{[103]}$ 

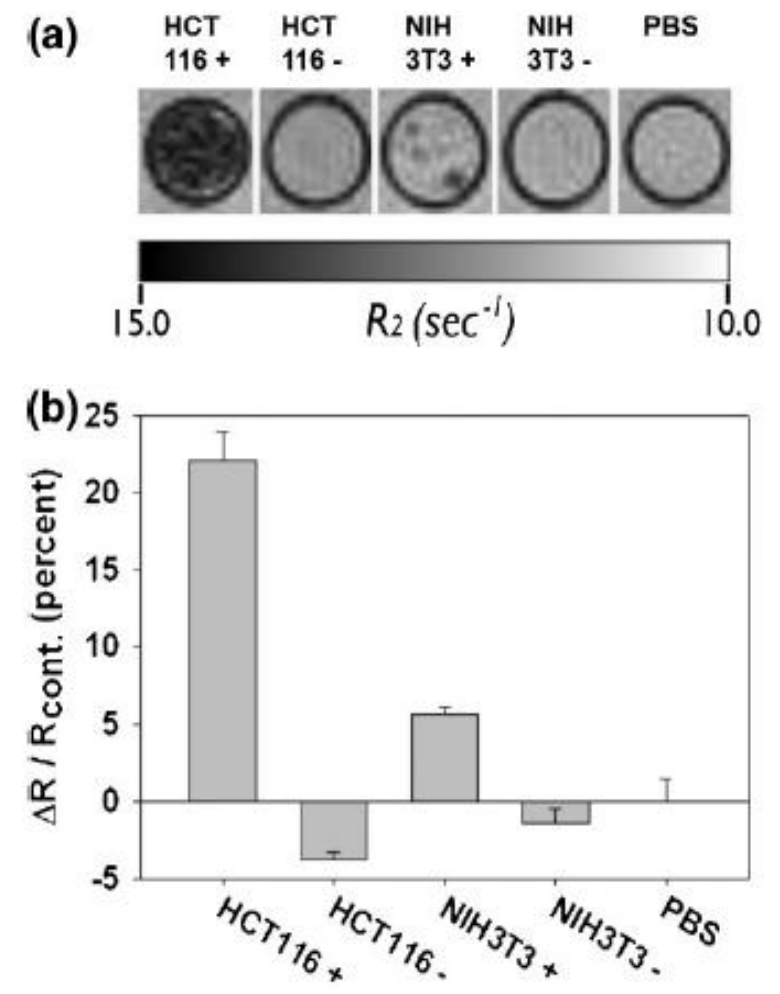

Figure 14. $\mathrm{T}_{2}$-weighted MR images and their color map for HCT116 and NIH3T3 cells (a) and relative relaxation rates $\left(R_{2}=\Delta R_{2} / R_{2 c o n t} ; R_{2}=T_{2}{ }^{-1}\right)(b)$. Notation: HCT116+: HA-SIONPs treated cells, HCT116-: control HCT116 cells, NIH3T3+: HA-SIONPs treated cells, and NIH3T3-: control NIH3T3 cells. Reprinted with permission from ref ${ }^{[103]}$. Copyright 2008 Wiley InterScience.

\subsection{Drug delivery}

Polymer-based drug delivery systems (Polymer-DDSs) have gained an increasing attention in polymer science, pharmaceutics, nanobiomedicine, and biomaterials science. In particular, polymer DDS conjugated with cell-targeting ligand biomolecules that can recognize specific cell receptors may enhance non-specificity of chemotherapeutic agents as well as reduce their side effects. Several types of polymer-DDS have been exploited, including polymer pro-drugs, ${ }^{[197]}$ micelles and vesicles based on amphiphilic and double block copolymers, ${ }^{[198,199]}$ dendrimers, ${ }^{[200]}$ hydrophobic polyester-based nanoparticulates, ${ }^{[201]}$ and microgels/nanogels. ${ }^{[151,202-204]}$ For in vivo drug delivery applications, several criteria are required for the design and development of effective polymer -DDS. Primary requirements 
include non-toxicity to cells, stability for prolonged circulation in blood stream, high loading efficiency, and controllable release of therapeutics. Additional requirements include biodegradability, novel functionality for further bioconjugation with cell-targeting biomolecules, and dimensional control. ${ }^{[205]}$

A recent advance in SIONP/polymer system is the development of SIONP-loaded polymer-DDS with cancer-cell targeting capability for controlled drug release and efficient MR imaging contrast characteristics. These systems can allow for real-time tumor-tracking by MR imaging upon controlled release of anticancer drugs in cancer cells. Self-assembled nanoparticles of amphiphilic block copolymers loaded with SIONPs and anticancer drugs such as Dox are typical examples for simultaneous drug delivery and MR imaging. Cell-targeting ligands such as folate, ${ }^{[128]}$ RGD tripeptide ${ }^{[127]}$ and antibody ${ }^{[125]}$ were attached to nanoparticles for intercellular delivery of anticancer drugs. They were then released upon collapse of micellar aggregates in cells after internalization into cells (Figure 15). PEG-based phospholipid self-assembled micelles consisting of SIONPs, quantum dots, and Dox were prepared for simultaneous magnetofluorecent imaging and drug delivery. ${ }^{[131]}$ 


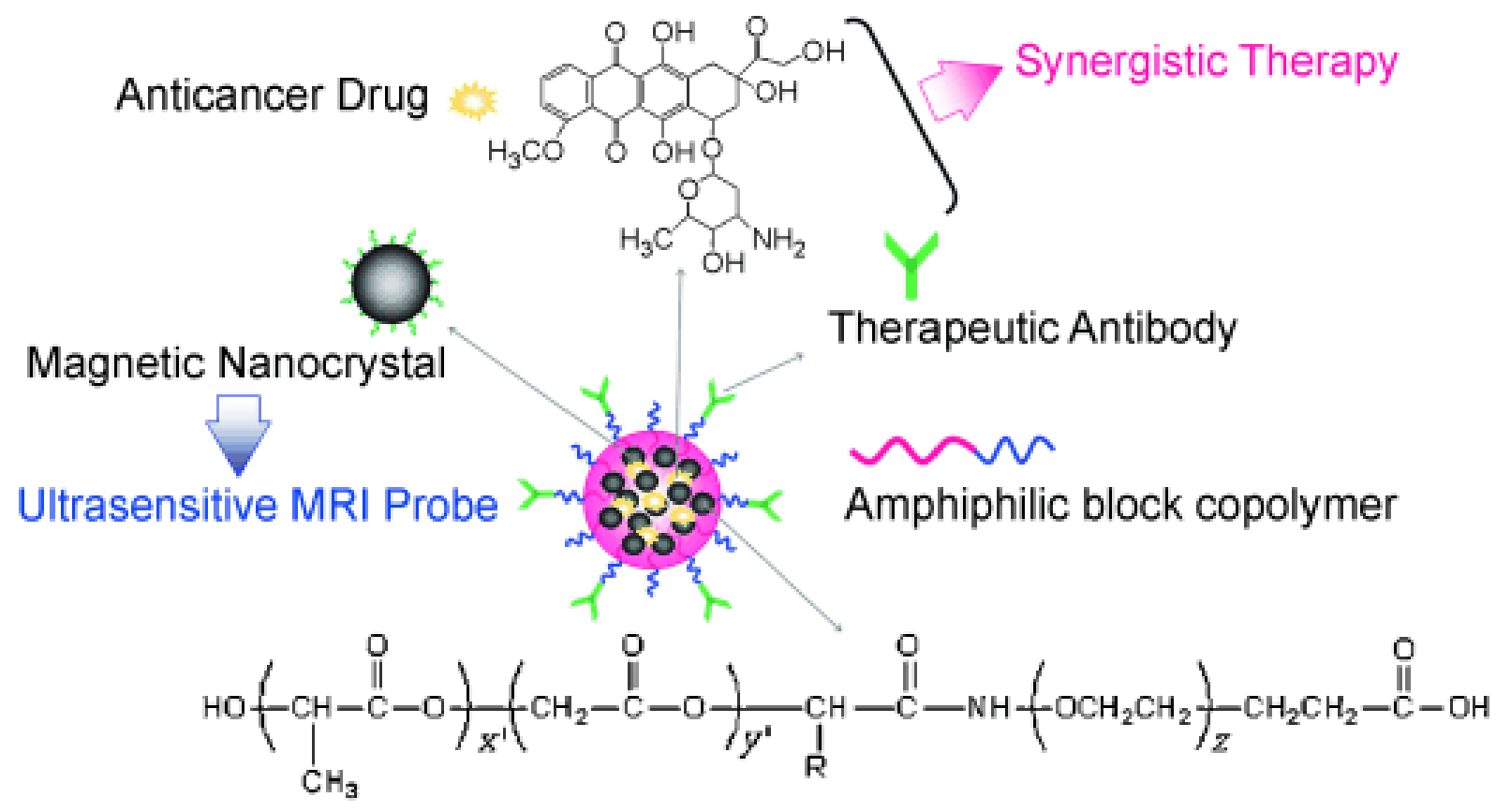

Figure 15. Schematic illustration for the fabrication of ultrasensitive MRI probe from core/shell micellar NPs consisting of hydrophobic PLA core embedded with SIONPs and Dox and hydrophilic PEO shell functionalized with targeting therapeutic antibodies to tumor. Reprinted with permission from ref ${ }^{[125]}$. Copyright 2007 Wiley InterScience.

Crosslinked microgels/nanogels/hydrogels embedded with SIONPs (ferrogels) have been designed for controllable release of drugs. In particular, ferrogels based on thermally-responsive polymers are attractive because temperature change is generated by applying an external magnetic field onto SIONPs. ${ }^{[206]}$ When magnetic field is on, temperature increased. At temperatures above lower critical solution temperature (LCST), thermoresponsive polymeric ferrogels are shrunken, enhancing release of drugs from ferrogels. When the magnetic field is absent and the temperature is below the LCST, they become swollen, reducing drug release. Therefore, a magnetically remote-controlled drug release can be achieved without additional stimuli. Ferrogels based on poly(vinyl alcohol ${ }^{[190]}$ and Fluronic PF127 triblock copolymer consisting of PEO and poly(propylene glycol) (PPO) were prepared by thermallyinduced sol-gel process. ${ }^{[189,207]}$ PF127 block copolymer formed micellar nanoparticles consisting of PPO core and PEO corona in water. In the presence of SIONPs and indomethacin (IMC), a hydrophobic 
drug, thermal gelation of the micelles occurred at elevated temperature, yielding ferrogels embedded with IMC which is mainly located in micelle cores. As seen Figure 16, the half-time $\left(\mathrm{t}_{1 / 2}\right)$ of drug release was reduced to $1500 \mathrm{~min}$ when magnetic field is on, compared to the $3195 \mathrm{~min}$ when magnetic field is off, indicating that the drug release is enhanced upon applying magnetic field. ${ }^{[207]}$ In addition, microgels/nanogels of water-soluble POEOMA, in which SIONPs are physically or covalently embedded, were prepared for drug delivery applications. ${ }^{[208,209]}$

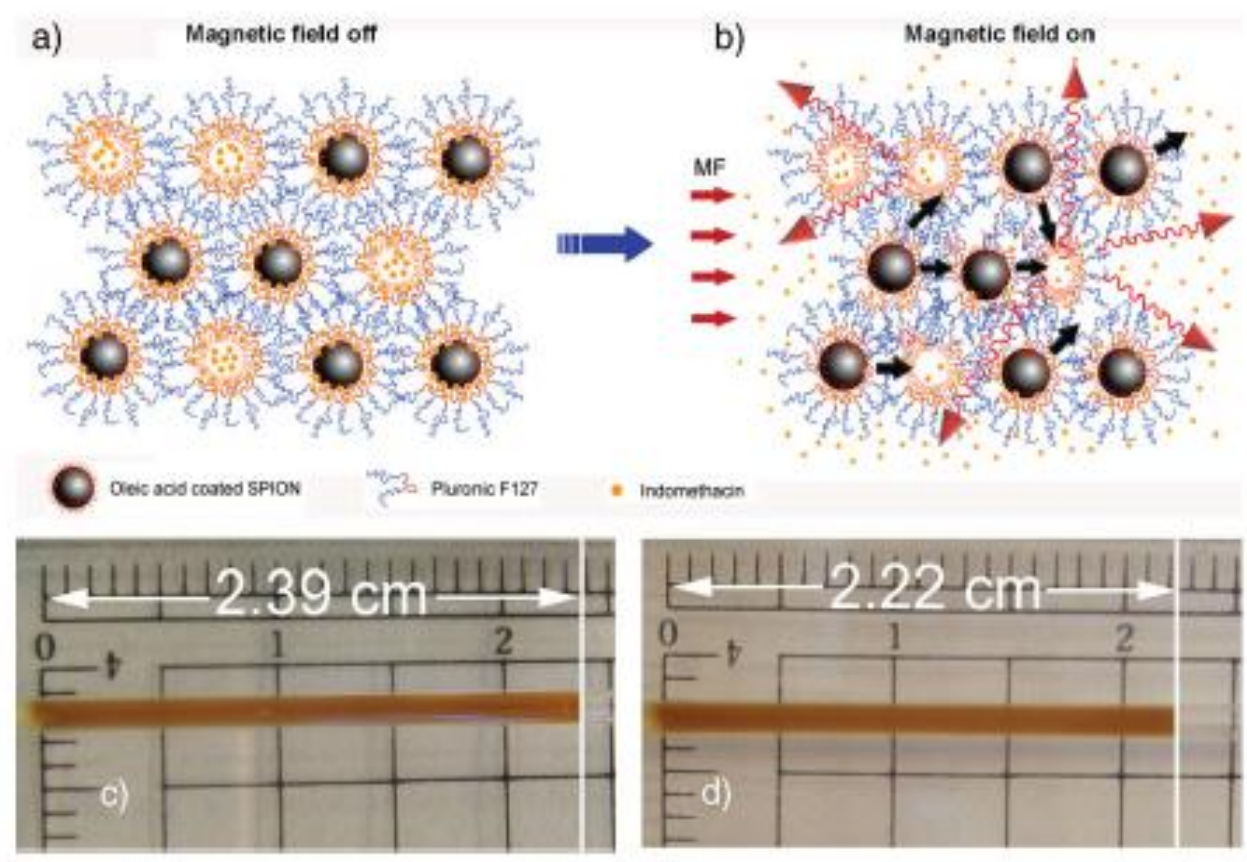

Figure 16. Schematic illustration of ordered microstructure of thermally-induced ferrogels based on Fluronic PF124 block copolymers: before applying magnetic field, IMG hydrophobic drug molecules are encapsulated in the hydrophobic core of micelles (a) and when magnetic field is on, SPONPs orient and approach each other, squeezing the micelles and leading to enhancement of IMC release (b). Reprinted with permission from ref ${ }^{\text {[207] }}$. Copyright 2009 Wiley InterScience.

\subsection{Other applications including hyperthermia, protein immobilization, and catalysts}

Hyperthermia therapy with SIONPs involves a local increase in temperature (up to $45^{\circ} \mathrm{C}$ ) when external magnetic field is applied on SIONPs. Such a temperature increase enables to kill temperaturesensitive cells, such as cancer cells. Recent studies using calorimetry shows that the heating rate 
depends on the particle size of SIONPs, in good agreement with theoretical prediction. This result suggests that the SIONPs should be designed to have optimal particle size with narrow size distribution for enhanced hyperthermia therapy. ${ }^{[210]}$ Several papers reported novel SIONP-polymer nanocomposites for hyperthermia. They include SIONPs coated with temperature-responsive PNIPAM-based copolymer $^{[92,184]}$ and chitosan ${ }^{[211]}$ as well as SIONPs embedded in hydrogels, ${ }^{[130,212,213]}$ solidified gels, ${ }^{[214]}$ and silica microparticles. ${ }^{[215]}$ In addition, well-designed polymer/SIONPs nanohybrids have been utilized for microfluidic separation, ${ }^{[105]}$ immobilization of proteins such as bovin serum albumin, ${ }^{[216]}$ and peroxidase-like catalyst. ${ }^{[105]}$

\section{Conclusion}

SIONPs have great potential for various biomedical applications, including MRI contrast enhancement, targeted drug delivery, hyperthermia, catalysis, biological separation, biosensors, and diagnostic medical devices. Polymers as multidentate ligands enhance the stability of SIONPs in solution as well as their optical, magnetic, and electronic properties. Various methods have been developed to yield unique polymer-SIONP hybrid nanomaterials. They include direct modification with polymers, surfaceinitiated controlled polymerization, inorganic silica/polymer hybridization, self-assembly and selfassociation, and various heterogeneous polymerization methods. The resulting hybrid magnetic polymer composites exhibit various morphologies such as magnetic core-polymer shell, magnetic multicores homogeneously dispersed in polymer matrix, raspberry morphology, and hair-like morphology. Direct modification with polymers requires the design, preparation, and modification of novel polymers including synthetic polymers and biopolymers. The general approaches include physical adsorption of well-controlled polymers, addition of second layer consisting of amphiphilic block copolymers, functional silica coating, and the ionic interaction approaches, yielding water-soluble/water-dispersible polymer-stabilized SIONPs. The surface-initiated CRP methods including ATRP and RAFT methods 
have been utilized for modification of single SIONP with well-controlled polymers. Inorganic silica/polymer hybridization involves the encapsulation of SIONPs with a silica shell through a sol-gel process of tetraethyl orthosilicate (TEOS). The silica-coated SIONPs are further functionalized and encapsulated with polymers, yielding multifunctional hybrid nanomaterials. Self-assembly method involves the assembly of amphiphilic block copolymers in water, forming stable core/shell micellar particles. The hydrophobic core serves as a carrier for SIONPs and anticancer drugs and the hydrophilic shell allows particle stabilization in aqueous solution. The amphiphilic block copolymers include biodegradable polyester-based amphiphilic block copolymers of PLA and PCL. Self-association method involves the physical association between SIONPs and anionic-or cationic polymers. The associations are typically achieved through ionic interaction, stereo-complexation, and sol-gel process with thermoresponsiveness. In addition, various heterogeneous polymerization methods of hydrophilic or water-soluble monomers have been extensively explored to prepare well-defined SIONP-embedded magnetic polymer spheres as well as crosslinked microgels/nanogels and hydrogels for biomedical applications. They include inverse (mini)emulsion polymerization, dispersion polymerization, precipitation polymerization, and bulk physical and chemical crosslinking. These methods have allowed for the preparation of noble hybrid magnetic polymer nanomaterials embedded with SIONPs for biorelated applications such as MR imaging (or dual imaging with optical imaging based on fluorescence), targeted drug delivery, hypothermia, protein immobilization, and biosensors.

Future design and development of effective magnetic polymer nanocomposites for biomedical applications require a higher degree of control over their key characteristics. Proper particle diameters in usually sub-micron size range can offer high surface areas for immobilization of biomolecules. Narrow size distribution can allow a uniform response to an external magnetic field. Appropriate surface functionalities enable selective adsorption or binding with biomolecules. Homogeneous distribution and proper encapsulation ensure high degree of non-toxicity and biocompatibility by avoiding direct contact 
of magnetic materials with some sensitive molecules and biomolecules (e.g. enzymes). Biodegradability and stimuli-responsiveness allow for controlled loading and release of encapsulated anticancer drugs and SIONPs. In addition, good colloidal stability in aqueous medium and high iron oxide content for rapid separation in the magnetic field are necessary.

\section{References}

[1] Cushing BL, Kolesnichenko VL, O'Connor CJ. Recent Advances in the Liquid-Phase Syntheses of Inorganic Nanoparticles. Chem. Rev. (Washington, D. C.) 2004;104:3893-946.

[2] Mornet S, Vasseur S, Grasset F, Duguet E. Magnetic nanoparticle design for medical diagnosis and therapy. Journal of Materials Chemistry 2004;14:2161-75.

[3] Gupta Ajay K, Wells S. Surface-modified superparamagnetic nanoparticles for drug delivery: preparation, characterization, and cytotoxicity studies. IEEE trans. nanobiosci. 2004;3:66-73.

[4] Sun C, Lee JSH, Zhang M. Magnetic nanoparticles in MR imaging and drug delivery. Adv. Drug Delivery Rev. 2008;60:1252-65.

[5] McCarthy JR, Weissleder R. Multifunctional magnetic nanoparticles for targeted imaging and therapy. Adv. Drug Delivery Rev. 2008;60:1241-51.

[6] Salgueirino-Maceira V, Correa-Duarte MA. Increasing the complexity of magnetic core/shell structured nanocomposites for biological applications. Advanced Materials 2007;19:4131-44.

[7] Gupta AK, Naregalkar RR, Vaidya VD, Gupta M. Recent advances on surface engineering of magnetic iron oxide nanoparticles and their biomedical applications. Nanomedicine 2007;2:2339.

[8] Veiseh O, Gunn JW, Zhang M. Design and fabrication of magnetic nanoparticles for targeted drug delivery and imaging. Adv. Drug Delivery Rev. 2010;62:284-304.

[9] Black CT, Murray CB, Sandstrom RL, Sun S. Spin-dependent tunneling in self-assembled cobalt-nanocrystal superlattices. Science 2000;290:1131-4.

[10] Yan Q, Purkayastha A, Kim T, Kroger R, Bose A, Ramanath G. Synthesis and assembly of monodisperse high-coercivity silica-capped FePt nanomagnets of tunable size, composition, and thermal stability from microemulsions. Advanced Materials 2006;18:2569-73.

[11] Gao J, Li L, Ho P-L, Mak GC, Gu H, Xu B. Combining fluorescent probes and biofunctional magnetic nanoparticles for rapid detection of bacteria in human blood. Advanced Materials 2006;18:3145-8.

[12] Gu H, Ho P-L, Tsang KWT, Wang L, Xu B. Using Biofunctional Magnetic Nanoparticles to Capture Vancomycin-Resistant Enterococci and Other Gram-Positive Bacteria at Ultralow Concentration. J. Amer. Chem. Soc. 2003;125:15702-3.

[13] Chen M, Liu JP, Sun S. One-Step Synthesis of FePt Nanoparticles with Tunable Size. J. Amer. Chem. Soc. 2004;126:8394-5.

[14] Kim J, Rong C, Liu JP, Sun S. Dispersible ferromagnetic FePt nanoparticles. Advanced Materials 2009;21:906-9.

[15] Srivastava S, Samanta B, Jordan BJ, Hong R, Xiao Q, Tuominen MT, et al. Integrated Magnetic Bionanocomposites through Nanoparticle-Mediated Assembly of Ferritin. J. Amer. Chem. Soc. 2007;129:11776-80. 
[16] Hou Y, Kondoh H, Kogure T, Ohta T. Preparation and Characterization of Monodisperse FePd Nanoparticles. Chemistry of Materials 2004;16:5149-52.

[17] Tzitzios V, Niarchos D, Margariti G, Fidler J, Petridis D. Synthesis of CoPt nanoparticles by a modified polyol method: characterization and magnetic properties. Nanotechnology 2005;16:287-91.

[18] Wang Y, Yang H. Synthesis of CoPt nanorods in ionic liquids. J. Am. Chem. Soc. 2005;127:5316-7.

[19] Ghosh M, Sampathkumaran EV, Rao CNR. Synthesis and Magnetic Properties of CoO Nanoparticles. Chemistry of Materials 2005;17:2348-52.

[20] Scarberry KE, Dickerson EB, McDonald JF, Zhang ZJ. Magnetic Nanoparticle-Peptide Conjugates for in Vitro and in Vivo Targeting and Extraction of Cancer Cells. J. Am. Chem. Soc. 2008;130:10258-62.

[21] Lee DC, Ghezelbash A, Stowell CA, Korgel BA. Synthesis and Magnetic Properties of Colloidal MnPt3 Nanocrystals. Journal of Physical Chemistry B 2006;110:20906-11.

[22] Taylor KML, Kim JS, Rieter WJ, An H, Lin W, Lin W. Mesoporous Silica Nanospheres as Highly Efficient MRI Contrast Agents. J. Amer. Chem. Soc. 2008;130:2154-5.

[23] Rowe MD, Chang C-C, Thamm DH, Kraft SL, Harmon Jr JF, Vogt AP, et al. Tuning the Magnetic Resonance Imaging Properties of Positive Contrast Agent Nanoparticles by Surface Modification with RAFT Polymers. Langmuir 2009;25:9487-99.

[24] Kim JS, Rieter WJ, Taylor KML, An H, Lin W, Lin W. Self-Assembled Hybrid Nanoparticles for Cancer-Specific Multimodal Imaging. J. Amer. Chem. Soc. 2007;129:8962-3.

[25] Cheng Z, Thorek DLJ, Tsourkas A. Gadolinium-Conjugated Dendrimer Nanoclusters as a Tumor-Targeted T1 Magnetic Resonance Imaging Contrast Agent. Angew. Chem. Int. Ed. 49:346-50.

[26] Crossley EL, Aitken JB, Vogt S, Harris HH, Rendina LM. Selective Aggregation of a PlatinumGadolinium Complex Within a Tumor-Cell Nucleus. Angew. Chem. Int. Ed. 2010;49:1231-3, $\mathrm{S} / 1-\mathrm{S} / 21$.

[27] Gao J, Zhang B, Gao Y, Pan Y, Zhang X, Xu B. Fluorescent Magnetic Nanocrystals by Sequential Addition of Reagents in a One-Pot Reaction: A Simple Preparation for Multifunctional Nanostructures. J. Am. Chem. Soc. 2007;129:11928-35.

[28] Choi J-s, Jun Y-w, Yeon S-I, Kim HC, Shin J-S, Cheon J. Biocompatible Heterostructured Nanoparticles for Multimodal Biological Detection. J. Am. Chem. Soc. 2006;128:15982-3.

[29] Kim H, Achermann M, Balet LP, Hollingsworth JA, Klimov VI. Synthesis and Characterization of Co/CdSe Core/Shell Nanocomposites: Bifunctional Magnetic-Optical Nanocrystals. J. Am. Chem. Soc. 2005;127:544-6.

[30] Sun X, Tabakman SM, Seo W-S, Zhang L, Zhang G, Sherlock S, et al. Separation of nanoparticles in a density gradient: FeCo@C and gold nanocrystals. Angew. Chem. Int. Ed. 2009;48:939-42.

[31] Yang Y, Chen O, Angerhofer A, Cao YC. On Doping CdS/ZnS Core/Shell Nanocrystals with Mn. J. Am. Chem. Soc. 2008;130:15649-61.

[32] Zhou T, Lu M, Zhang Z, Gong H, Chin WS, Liu B. Synthesis and Characterization of Multifunctional FePt/ZnO Core/Shell Nanoparticles. Advanced Materials 2010;22:403-6.

[33] Lee Y, Garcia MA, Frey Huls NA, Sun S. Synthetic Tuning of the Catalytic Properties of AuFe3O4 Nanoparticles. Angew. Chem. Int. Ed. 2010;49:1271-4, S/1-S/4.

[34] Xu C, Xu K, Gu H, Zheng R, Liu H, Zhang X, et al. Dopamine as a robust anchor to immobilize functional molecules on the iron oxide shell of magnetic nanoparticles. J. Amer. Chem. Soc. 2004;126:9938-9. 
[35] Bertorelle F, Wilhelm C, Roger J, Gazeau F, Menager C, Cabuil V. Fluorescence-Modified Superparamagnetic Nanoparticles: Intracellular Uptake and Use in Cellular Imaging. Langmuir 2006;22:5385-91.

[36] Kim T, Reis L, Rajan K, Shima M. Magnetic behavior of iron oxide nanoparticle-biomolecule assembly. Journal of Magnetism and Magnetic Materials 2005;295:132-8.

[37] Thomas LA, Dekker L, Kallumadil M, Southern P, Wilson M, Nair SP, et al. Carboxylic acidstabilized iron oxide nanoparticles for use in magnetic hyperthermia. Journal of Materials Chemistry 2009;19:6529-35.

[38] Basly B, Felder-Flesch D, Perriat P, Billotey C, Taleb J, Pourroy G, et al. Dendronized iron oxide nanoparticles as contrast agents for MRI. Chemical Communications (Cambridge) 2010;46:985-7.

[39] Bucak S, Jones DA, Laibinis PE, Hatton TA. Protein Separations Using Colloidal Magnetic Nanoparticles. Biotechnol. Prog. 2003;19:477-84.

[40] Shen L, Laibinis PE, Hatton TA. Bilayer Surfactant Stabilized Magnetic Fluids: Synthesis and Interactions at Interfaces. Langmuir 1999;15:447-53.

[41] Shen L, Stachowiak A, Hatton TA, Laibinis PE. Polymerization of Olefin-Terminated Surfactant Bilayers on Magnetic Fluid Nanoparticles. Langmuir 2000;16:9907-11.

[42] Boal AK, Das K, Gray M, Rotello VM. Monolayer Exchange Chemistry of g-Fe2O3 Nanoparticles. Chemistry of Materials 2002;14:2628-36.

[43] Song H-T, Choi J-s, Huh Y-M, Kim S, Jun Y-w, Suh J-S, et al. Surface Modulation of Magnetic Nanocrystals in the Development of Highly Efficient Magnetic Resonance Probes for Intracellular Labeling. J. Amer. Chem. Soc. 2005;127:9992-3.

[44] Huh Y-M, Jun Y-W, Song H-T, Kim S, Choi J-S, Lee J-H, et al. In Vivo Magnetic Resonance Detection of Cancer by Using Multifunctional Magnetic Nanocrystals. J. Amer. Chem. Soc. 2005;127:12387-91.

[45] Fried T, Shemer G, Markovich G. Ordered two-dimensional arrays of ferrite nanoparticles. Advanced Materials 2001;13:1158-61.

[46] Kohler N, Sun C, Fichtenholtz A, Gunn J, Fang C, Zhang M. Methotrexate-immobilized poly(ethylene glycol) magnetic nanoparticles for MR imaging and drug delivery. Small 2006;2:785-92.

[47] Kohler N, Fryxell GE, Zhang M. A Bifunctional Poly(ethylene glycol) Silane Immobilized on Metallic Oxide-Based Nanoparticles for Conjugation with Cell Targeting Agents. J. Amer. Chem. Soc. 2004;126:7206-11.

[48] Kohler N, Sun C, Wang J, Zhang M. Methotrexate-Modified Superparamagnetic Nanoparticles and Their Intracellular Uptake into Human Cancer Cells. Langmuir 2005;21:8858-64.

[49] El-Boubbou K, Gruden C, Huang X. Magnetic Glyco-nanoparticles: A Unique Tool for Rapid Pathogen Detection, Decontamination, and Strain Differentiation. J. Amer. Chem. Soc. 2007;129:13392-3.

[50] El-Boubbou K, Zhu DC, Vasileiou C, Borhan B, Prosperi D, Li W, et al. Magnetic GlycoNanoparticles: A Tool To Detect, Differentiate, and Unlock the Glyco-Codes of Cancer via Magnetic Resonance Imaging. J. Amer. Chem. Soc. 2010;132:4490-9.

[51] Bergemann C, Muller-Schulte D, Oster J, a Brassard L, Lubbe AS. Magnetic ion-exchange nano- and microparticles for medical, biochemical and molecular biological applications. Journal of Magnetism and Magnetic Materials 1999;194:45-52.

[52] Horak D, Babic M, Jendelova P, Herynek V, Trchova M, Pientka Z, et al. D-Mannose-Modified Iron Oxide Nanoparticles for Stem Cell Labeling. Bioconjugate Chem. 2007;18:635-44.

[53] Li Z, Chen H, Bao H, Gao M. One-Pot Reaction to Synthesize Water-Soluble Magnetite Nanocrystals. Chemistry of Materials 2004;16:1391-3. 
[54] Hu F, Wei L, Zhou Z, Ran Y, Li Z, Gao M. Preparation of biocompatible magnetite nanocrystals for in vivo magnetic resonance detection of cancer. Advanced Materials 2006;18:2553-6.

[55] Park J-H, von Maltzahn G, Zhang L, Schwartz MP, Ruoslahti E, Bhatia SN, et al. Magnetic iron oxide nanoworms for tumor targeting and imaging. Advanced Materials 2008;20:1630-5.

[56] Tomczak N, Janczewski D, Han M, Vancso GJ. Designer polymer-quantum dot architectures. Progress in Polymer Science 2009;34:393-430.

[57] Horak D, Babic M, Mackova H, Benes MJ. Preparation and properties of magnetic nano- and microsized particles for biological and environmental separations. Journal of Separation Science 2007;30:1751-72.

[58] Frenz L, El Harrak A, Pauly M, Begin-Colin S, Griffiths AD, Baret J-C. Droplet-based microreactors for the synthesis of magnetic iron oxide nanoparticles. Angew. Chem. Int. Ed. 2008;47:6817-20.

[59] Rockenberger J, Scher EC, Alivisatos AP. A New Nonhydrolytic Single-Precursor Approach to Surfactant-Capped Nanocrystals of Transition Metal Oxides. J. Amer. Chem. Soc. 1999;121:11595-6.

[60] Hyeon T, Lee SS, Park J, Chung Y, Na HB. Synthesis of Highly Crystalline and Monodisperse Maghemite Nanocrystallites without a Size-Selection Process. J. Amer. Chem. Soc. 2001;123:12798-801.

[61] Sun S, Zeng H. Size-Controlled Synthesis of Magnetite Nanoparticles. J. Amer. Chem. Soc. 2002;124:8204-5.

[62] Woo K, Hong J, Choi S, Lee H-W, Ahn J-P, Kim CS, et al. Easy Synthesis and Magnetic Properties of Iron Oxide Nanoparticles. Chemistry of Materials 2004;16:2814-8.

[63] Kovalenko MV, Bodnarchuk MI, Lechner RT, Hesser G, Schaeffler F, Heiss W. Fatty Acid Salts as Stabilizers in Size- and Shape-Controlled Nanocrystal Synthesis: The Case of Inverse Spinel Iron Oxide. J. Amer. Chem. Soc. 2007;129:6352-3.

[64] Peng S, Sun S. Synthesis and characterization of monodisperse hollow Fe3O4 nanoparticles. Angew. Chem. Int. Ed. 2007;46:4155-8.

[65] Pinna N, Grancharov S, Beato P, Bonville P, Antonietti M, Niederberger M. Magnetite Nanocrystals: Nonaqueous Synthesis, Characterization, and Solubility. Chemistry of Materials 2005; 17:3044-9.

[66] Deng H, Li X, Peng Q, Wang X, Chen J, Li Y. Monodisperse magnetic single-crystal ferrite microspheres. Angew. Chem. Int. Ed. 2005;44:2782-5.

[67] Bai F, Wang D, Huo Z, Chen W, Liu L, Liang X, et al. A versatile bottom-up assembly approach to colloidal spheres from nanocrystals. Angew. Chem. Int. Ed. 2007;46:6650-3.

[68] Liu J, Sun Z, Deng Y, Zou Y, Li C, Guo X, et al. Highly Water-Dispersible Biocompatible Magnetite Particles with Low Cytotoxicity Stabilized by Citrate Groups. Angew. Chem., Int. Ed. 2009;48:5875-9, S/1-S/16.

[69] Ge J, Hu Y, Biasini M, Beyermann WP, Yin Y. Superparamagnetic magnetite colloidal nanocrystal clusters. Angew. Chem. Int. Ed. 2007;46:4342-5.

[70] Jia C-J, Sun L-D, Luo F, Han X-D, Heyderman LJ, Yan Z-G, et al. Large-Scale Synthesis of Single-Crystalline Iron Oxide Magnetic Nanorings. J. Amer. Chem. Soc. 2008;130:16968-77.

[71] Wang D, He J, Rosenzweig N, Rosenzweig Z. Superparamagnetic Fe2O3 Beads-CdSe/ZnS Quantum Dots Core-Shell Nanocomposite Particles for Cell Separation. Nano Letters 2004;4:409-13.

[72] Lim J, Eggeman A, Lanni F, Tilton RD, Majetich SA. Synthesis and single-particle optical detection of low-polydispersity plasmonic-superparamagnetic nanoparticles. Advanced Materials 2008;20:1721-6. 
[73] Nash MA, Lai JJ, Hoffman AS, Yager P, Stayton PS. "Smart" Diblock Copolymers as Templates for Magnetic-Core Gold-Shell Nanoparticle Synthesis. Nano Letters 2010;10:85-91.

[74] Gao J, Liang G, Cheung JS, Pan Y, Kuang Y, Zhao F, et al. Multifunctional Yolk-Shell Nanoparticles: A Potential MRI Contrast and Anticancer Agent. J. Amer. Chem. Soc. 2008;130:11828-33.

[75] Gao J, Zhang W, Huang P, Zhang B, Zhang X, Xu B. Intracellular Spatial Control of Fluorescent Magnetic Nanoparticles. J. Am. Chem. Soc. 2008;130:3710-1.

[76] Park J-H, von Maltzahn G, Ruoslahti E, Bhatia SN, Sailor MJ. Micellar hybrid nanoparticles for simultaneous magnetofluorescent imaging and drug delivery. Angew. Chem., Int. Ed. 2008;47:7284-8.

[77] Li L, Feng Y, Li Y, Zhao W, Shi J. Fe3O4 Core/Layered Double Hydroxide Shell Nanocomposite: Versatile Magnetic Matrix for Anionic Functional Materials. Angew. Chem. Int. Ed. 2009;48:5888-92, S/1-S/1.

[78] Jang J-t, Nah H, Lee J-H, Moon SH, Kim MG, Cheon J. Critical enhancements of MRI contrast and hyperthermic effects by dopant-controlled magnetic nanoparticles. Angew. Chem. Int. Ed. 2009;48:1234-8.

[79] Lee J-H, Lee K, Moon SH, Lee Y, Park TG, Cheon J. All-in-One Target-Cell-Specific Magnetic Nanoparticles for Simultaneous Molecular Imaging and siRNA Delivery. Angew. Chem. Int. Ed. 2009;48:4174-9, S/1-S/5.

[80] Zeng H, Rice PM, Wang SX, Sun S. Shape-Controlled Synthesis and Shape-Induced Texture of MnFe2O4 Nanoparticles. J. Amer. Chem. Soc. 2004;126:11458-9.

[81] Xuan Y, Li Q, Yang G. Synthesis and magnetic properties of Mn-Zn ferrite nanoparticles. Journal of Magnetism and Magnetic Materials 2007;312:464-9.

[82] Lu Y, Yin Y, Mayers BT, Xia Y. Modifying the surface properties of superparamagnetic iron oxide nanoparticles through a sol-gel approach. Nano Letters 2002;2:183-6.

[83] Kim J, Lee JE, Lee J, Yu JH, Kim BC, An K, et al. Magnetic Fluorescent Delivery Vehicle Using Uniform Mesoporous Silica Spheres Embedded with Monodisperse Magnetic and Semiconductor Nanocrystals. J. Amer. Chem. Soc. 2006;128:688-9.

[84] Yi DK, Selvan ST, Lee SS, Papaefthymiou GC, Kundaliya D, Ying JY. Silica-Coated Nanocomposites of Magnetic Nanoparticles and Quantum Dots. J. Amer. Chem. Soc. 2005;127:4990-1.

[85] Zhao W, Gu J, Zhang L, Chen H, Shi J. Fabrication of uniform magnetic nanocomposite spheres with a magnetic core/mesoporous silica shell structure. J. Amer. Chem. Soc. 2005;127:8916-7.

[86] Hu S-H, Chen S-Y, Liu D-M, Hsiao C-S. Core/single-crystal-shell nanospheres for controlled drug release via a magnetically triggered rupturing mechanism. Advanced Materials 2008;20:2690-5.

[87] Zhao N, Gao M. Magnetic Janus particles prepared by a flame synthetic approach: synthesis, characterizations and properties. Advanced Materials 2009;21:184-7.

[88] Lee J-H, Jun Y-w, Yeon S-I, Shin J-S, Cheon J. Dual-mode nanoparticle probes for highperformance magnetic resonance and fluorescence imaging of neuroblastoma. Angew. Chem. Int. Ed. 2006;45:8160-2.

[89] Matyjaszewski K, Davis TP, John Wiley \& Sons Inc., 2002.

[90] Lutz J-F, Stiller S, Hoth A, Kaufner L, Pison U, Cartier R. One-Pot Synthesis of PEGylated Ultrasmall Iron-Oxide Nanoparticles and Their in Vivo Evaluation as Magnetic Resonance Imaging Contrast Agents. Biomacromolecules 2006;7:3132-8.

[91] Zhang Q, Wang C, Qiao L, Yan H, Liu K. Superparamagnetic iron oxide nanoparticles coated with a folate-conjugated polymer. Journal of Materials Chemistry 2009;19:8393-402. 
[92] Aqil A, Vasseur S, Duguet E, Passirani C, Benoit JP, Jerome R, et al. Magnetic nanoparticles coated by temperature responsive copolymers for hyperthermia. Journal of Materials Chemistry 2008;18:3352-60.

[93] Papaphilippou P, Loizou L, Popa NC, Han A, Vekas L, Odysseos A, et al. Superparamagnetic Hybrid Micelles, Based on Iron Oxide Nanoparticles and Well-Defined Diblock Copolymers Possessing b-Ketoester Functionalities. Biomacromolecules 2009;10:2662-71.

[94] Underhill RS, Liu G. Triblock nanospheres and their use as templates for inorganic nanoparticle preparation. Chemistry of Materials 2000;12:2082-91.

[95] Harris LA, Goff JD, Carmichael AY, Riffle JS, Harburn JJ, St. Pierre TG, et al. Magnetite nanoparticle dispersions stabilized with triblock copolymers. Chemistry of Materials 2003;15:1367-77.

[96] Kumagai M, Kano MR, Morishita Y, Ota M, Imai Y, Nishiyama N, et al. Enhanced magnetic resonance imaging of experimental pancreatic tumor in vivo by block copolymer-coated magnetite nanoparticles with TGF-b inhibitor. J. Controlled Release 2009;140:306-11.

[97] Biswas S, Belfield KD, Das RK, Ghosh S, Hebard AF. Block Copolymer-Mediated Formation of Superparamagnetic Nanocomposites. Chemistry of Materials 2009;21:5644-53.

[98] Qin J, Laurent S, Jo YS, Roch A, Mikhaylova M, Bhujwalla ZM, et al. A high-performance magnetic resonance imaging T2 contrast agent. Advanced Materials 2007;19:1874-8.

[99] Qin J, Jo YS, Muhammed M. Coating Nanocrystals with Amphiphilic Thermosensitive Copolymers. Angew. Chem. Int. Ed. 2009;48:7845-9, S/1-S/5.

[100] Lee H, Lee E, Kim DK, Jang NK, Jeong YY, Jon S. Antibiofouling Polymer-Coated Superparamagnetic Iron Oxide Nanoparticles as Potential Magnetic Resonance Contrast Agents for in Vivo Cancer Imaging. J. Amer. Chem. Soc. 2006;128:7383-9.

[101] Lee H, Yu MK, Park S, Moon S, Min JJ, Jeong YY, et al. Thermally Cross-Linked Superparamagnetic Iron Oxide Nanoparticles: Synthesis and Application as a Dual Imaging Probe for Cancer in Vivo. J. Amer. Chem. Soc. 2007;129:12739-45.

[102] Babic M, Horak D, Trchova M, Jendelova P, Glogarova K, Lesny P, et al. Poly(L-lysine)modified iron oxide nanoparticles for stem cell labeling. Bioconjug Chem 19:740-50. FIELD Reference Number: FIELD Journal Code:9010319 FIELD Call Number:.

[103] Lee Y, Lee H, Kim YB, Kim J, Hyeon T, Park H, et al. Bioinspired surface immobilization of hyaluronic acid on monodisperse magnetite nanocrystals for targeted cancer imaging. Advanced Materials 2008;20:4154-7.

[104] Huh Y-M, Lee E-S, Lee J-H, Jun Y-w, Kim P-H, Yun C-O, et al. Hybrid nanoparticles for magnetic resonance imaging of target-specific viral gene delivery. Advanced Materials 2007;19:3109-12.

[105] Nath S, Kaittanis C, Ramachandran V, Dalal NS, Perez JM. Synthesis, Magnetic Characterization, and Sensing Applications of Novel Dextran-Coated Iron Oxide Nanorods. Chemistry of Materials 2009;21:1761-7.

[106] Lewin M, Carlesso N, Tung C-H, Tang X-W, Cory D, Scadden DT, et al. Tat peptidederivatized magnetic nanoparticles allow in vivo tracking and recovery of progenitor cells. Nature Biotechnology 2000;18:410-4.

[107] Xie J, Wang J, Niu G, Huang J, Chen K, Li X, et al. Human serum albumin coated iron oxide nanoparticles for efficient cell labeling. Chemical Communications (Cambridge) 2010;46:433-5.

[108] Bulte JWM, Douglas T, Witwer B, Zhang S-C, Strable E, Lewis BK, et al. Magnetodendrimers allow endosomal magnetic labeling and in vivo tracking of stem cells. Nat. Biotech. 2001;19:1141-7. 
[109] Vestal CR, Zhang ZJ. Atom Transfer Radical Polymerization Synthesis and Magnetic Characterization of MnFe2O4/Polystyrene Core/Shell Nanoparticles. J. Amer. Chem. Soc. 2002;124:14312-3.

[110] Fan Q-L, Neoh K-G, Kang E-T, Shuter B, Wang S-C. Solvent-free atom transfer radical polymerization for the preparation of poly(poly(ethyleneglycol) monomethacrylate)-grafted Fe3O4 nanoparticles: Synthesis, characterization and cellular uptake. Biomaterials 2007;28:5426-36.

[111] Wang Y, Teng X, Wang J-S, Yang H. Solvent-Free Atom Transfer Radical Polymerization in the Synthesis of Fe2O3 @ Polystyrene Core-Shell Nanoparticles. Nano Letters 2003;3:789-93.

[112] Li G, Fan J, Jiang R, Gao Y. Cross-linking the Linear Polymeric Chains in the ATRP Synthesis of Iron Oxide/Polystyrene Core/Shell Nanoparticles. Chemistry of Materials 2004;16:1835-7.

[113] Gelbrich T, Feyen M, Schmidt AM. Magnetic thermoresponsive core-shell nanoparticles. Macromolecules 2006;39:3469-72.

[114] Garcia I, Zafeiropoulos NE, Janke A, Tercjak A, Eceiza A, Stamm M, et al. Functionalization of iron oxide magnetic nanoparticles with poly(methyl methacrylate) brushes via grafting-from atom transfer radical polymerization. J. Polym. Sci. Part A Polym. Chem. 2007;45:925-32.

[115] Czaun M, Hevesi L, Takafuji M, Ihara H. A novel approach to magneto-responsive polymeric gels assisted by iron nanoparticles as nano cross-linkers. Chemical Communications (Cambridge) 2008:2124-6.

[116] Hu F, Neoh KG, Cen L, Kang E-T. Cellular Response to Magnetic Nanoparticles \"PEGylated\" via Surface-Initiated Atom Transfer Radical Polymerization. Biomacromolecules 2006;7:809-16.

[117] Wang W-C, Neoh K-G, Kang E-T. Surface functionalization of Fe3O4 magnetic nanoparticles via RAFT-mediated graft polymerization. Macromol. Rapid Commun. 2006;27:1665-9.

[118] Xiao Z-P, Yang K-M, Liang H, Lu J. Synthesis of magnetic, reactive, and thermoresponsive $\mathrm{Fe} 3 \mathrm{O} 4$ nanoparticles via surface-initiated RAFT copolymerization of $\mathrm{N}$-isopropylacrylamide and acrolein. J. Polym. Sci. Part A Polym. Chem. 2010;48:542-50.

[119] Saoud FM, Tonge MP, Weber WG, Sanderson RD. Magnetite Nanoparticles for the Preparation of Ultrapure RAFT Polymers. Macromolecules 2008;41:1598-600.

[120] Goff JD, Huffstetler PP, Miles WC, Pothayee N, Reinholz CM, Ball S, et al. Novel Phosphonate-Functional Poly(ethylene oxide)-Magnetite Nanoparticles Form Stable Colloidal Dispersions in Phosphate-Buffered Saline. Chemistry of Materials 2009;21:4784-95.

[121] Li G, Zeng DL, Wang L, Baoyu, Zong, Neoh KG, et al. Hairy Hybrid Nanoparticles of Magnetic Core, Fluorescent Silica Shell, and Functional Polymer Brushes. Macromolecules 2009;42:85615.

[122] Zhou J, Meng L, Lu Q, Fu J, Huang X. Superparamagnetic submicro-megranates: Fe3O4 nanoparticles coated with highly cross-linked organic/inorganic hybrids. Chemical Communications (Cambridge) 2009:6370-2.

[123] Liu C, Guo J, Yang W, Hu J, Wang C, Fu S. Magnetic mesoporous silica microspheres with thermo-sensitive polymer shell for controlled drug release. Journal of Materials Chemistry 2009;19:4764-70.

[124] Zhang F, Wang C-C. Preparation of P(NIPAM-co-AA) Microcontainers Surface-Anchored with Magnetic Nanoparticles. Langmuir 2009;25:8255-62.

[125] Yang J, Lee C-H, Ko H-J, Suh J-S, Yoon H-G, Lee K, et al. Multifunctional magneto-polymeric nanohybrids for targeted detection and synergistic therapeutic effects on breast cancer. Angew. Chem., Int. Ed. 2007;46:8836-9.

[126] Hu F, Neoh KG, Kang E-T. Synthesis of Folic Acid Functionalized PLLA-b-PPEGMA Nanoparticles for Cancer Cell Targeting. Macromol. Rapid Commun. 2009;30:609-14. 
[127] Nasongkla N, Bey E, Ren J, Ai H, Khemtong C, Guthi JS, et al. Multifunctional Polymeric Micelles as Cancer-Targeted, MRI-Ultrasensitive Drug Delivery Systems. Nano Letters 2006;6:2427-30.

[128] Yang X, Chen Y, Yuan R, Chen G, Blanco E, Gao J, et al. Folate-encoded and Fe3O4-loaded polymeric micelles for dual targeting of cancer cells. Polymer 2008;49:3477-85.

[129] Kim B-S, Qiu J-M, Wang J-P, Taton TA. Magnetomicelles: composite nanostructures from magnetic nanoparticles and cross-linked amphiphilic block copolymers. Nano Lett 2005;5:198791.

[130] Shi D, Cho HS, Chen Y, Xu H, Gu H, Lian J, et al. Fluorescent Polystyrene-Fe3O4 Composite Nanospheres for In Vivo Imaging and Hyperthermia. Advanced Materials 2009;21:2170-3.

[131] Park J-H, von Maltzahn G, Ruoslahti E, Bhatia SN, Sailor MJ. Micellar hybrid nanoparticles for simultaneous magnetofluorescent imaging and drug delivery. Angew. Chem. Int. Ed. 2008;47:7284-8.

[132] Frankamp BL, Boal AK, Tuominen MT, Rotello VM. Direct Control of the Magnetic Interaction between Iron Oxide Nanoparticles through Dendrimer-Mediated Self-Assembly. J. Amer. Chem. Soc. 2005;127:9731-5.

[133] Fresnais J, Berret JF, Frka-Petesic B, Sandre O, Perzynski R. Electrostatic co-assembly of iron oxide nanoparticles and polymers: towards the generation of highly persistent superparamagnetic nanorods. Advanced Materials 2008;20:3877-81.

[134] Liem KP, Mart RJ, Webb SJ. Magnetic Assembly and Patterning of Vesicle/Nanoparticle Aggregates. J. Amer. Chem. Soc. 2007;129:12080-1.

[135] Toprak MS, McKenna BJ, Mikhaylova M, Waite JH, Stucky GD. Spontaneous assembly of magnetic microspheres. Advanced Materials 2007;19:1362-8.

[136] Babic M, Horak D, Trchova M, Jendelova P, Glogarova K, Lesny P, et al. Poly(L-lysine)Modified Iron Oxide Nanoparticles for Stem Cell Labeling. Bioconjugate Chem. 2008;19:74050 .

[137] Hou Y, Kondoh H, Shimojo M, Sako EO, Ozaki N, Kogure T, et al. Inorganic Nanocrystal SelfAssembly via the Inclusion Interaction of b-Cyclodextrins: Toward 3D Spherical Magnetite. Journal of Physical Chemistry B 2005;109:4845-52.

[138] Okassa LN, Marchais H, Douziech-Eyrolles L, Herve K, Cohen-Jonathan S, Munnier E, et al. Optimization of iron oxide nanoparticles encapsulation within poly(D,L-lactide-co-glycolide) sub-micron particles. Eur. J. Pharm. Biopharm. 2007;67:31-8.

[139] Hamoudeh M, Fessi H. Preparation, characterization and surface study of poly-epsilon caprolactone magnetic microparticles. J Colloid Interface Sci 2006;300:584-90.

[140] Urban M, Musyanovych A, Landfester K. Fluorescent Superparamagnetic Polylactide Nanoparticles by Combination of Miniemulsion and Emulsion/Solvent Evaporation Techniques. Macromol. Chem. Phys. 2009;210:961-70.

[141] Yang C, Guan Y, Xing J, Liu H. Surface Functionalization and Characterization of Magnetic Polystyrene Microbeads. Langmuir 2008;24:9006-10.

[142] Cui L, Xu H, He P, Sumitomo K, Yamaguchi Y, Gu H. Developing a hybrid emulsion polymerization system to synthesize $\mathrm{Fe} 3 \mathrm{O} 4$ /polystyrene latexes with narrow size distribution and high magnetite content. J. Polym. Sci. Part A Polym. Chem. 2007;45:5285-95.

[143] Joumaa N, Toussay P, Lansalot M, Elaissari A. Surface modification of iron oxide nanoparticles by a phosphate-based macromonomer and further encapsulation into submicrometer polystyrene particles by miniemulsion polymerization. J. Polym. Sci. Part A Polym. Chem. 2007;46:327-40.

[144] Luo Y-D, Dai C-A, Chiu W-Y. Polystyrene/Fe3O4 composite latex via miniemulsion polymerization-nucleation mechanism and morphology. J. Polym. Sci. Part A Polym. Chem. 2008;46:1014-24. 
[145] Lu S, Ramos J, Forcada J. Self-Stabilized Magnetic Polymeric Composite Nanoparticles by Emulsifier-Free Miniemulsion Polymerization. Langmuir 2007;23:12893-900.

[146] Qian Z, Zhang Z, Chen Y. A novel preparation of surface-modified paramagnetic magnetite/polystyrene nanocomposite microspheres by radiation-induced miniemulsion polymerization. Journal of Colloid and Interface Science 2008;327:354-61.

[147] Xu X, Friedman G, Humfeld KD, Majetich SA, Asher SA. Synthesis and Utilization of Monodisperse Superparamagnetic Colloidal Particles for Magnetically Controllable Photonic Crystals. Chemistry of Materials 2002;14:1249-56.

[148] Xu X, Majetich SA, Asher SA. Mesoscopic Monodisperse Ferromagnetic Colloids Enable Magnetically Controlled Photonic Crystals. J. Amer. Chem. Soc. 2002;124:13864-8.

[149] Yang S, Liu H, Zhang Z. A facile route to hollow superparamagnetic magnetite/polystyrene nanocomposite microspheres via inverse miniemulsion polymerization. J. Polym. Sci. Part A Polym. Chem. 2008;46:3900-10.

[150] Antonietti M, Landfester K. Polyreactions in miniemulsions. Progress in Polymer Science 2002;27:689-757.

[151] Oh JK, Drumright R, Siegwart DJ, Matyjaszewski K. The development of microgels/nanogels for drug delivery applications. Progress in Polymer Science 2008;33:448-77.

[152] Kriwet B, Walter E, Kissel T. Synthesis of bioadhesive poly(acrylic acid) nano- and microparticles using an inverse emulsion polymerization method for the entrapment of hydrophilic drug candidates. J. Controlled Release 1998;56:149-58.

[153] Landfester K, Willert M, Antonietti M. Preparation of polymer particles in nonaqueous direct and inverse miniemulsions. Macromolecules 2000;33:2370-6.

[154] Sun Q, Deng Y. In Situ Synthesis of Temperature-Sensitive Hollow Microspheres via Interfacial Polymerization. J. Amer. Chem. Soc. 2005;127:8274-5.

[155] Owens DE, III, Jian Y, Fang JE, Slaughter BV, Chen Y-H, Peppas NA. Thermally responsive swelling properties of polyacrylamide/poly(acrylic acid) interpenetrating polymer network nanoparticles. Macromolecules 2007;40:7306-10.

[156] Missirlis D, Tirelli N, Hubbell JA. Amphiphilic Hydrogel Nanoparticles. Preparation, Characterization, and Preliminary Assessment as New Colloidal Drug Carriers. Langmuir 2005;21:2605-13.

[157] Gao D, Xu H, Philbert MA, Kopelman R. Bioeliminable Nanohydrogels for Drug Delivery. Nano Letters 2008;8:3320-4.

[158] Gaur U, Sahoo SK, De TK, Ghosh PC, Maitra A, Ghosh PK. Biodistribution of fluoresceinated dextran using novel nanoparticles evading reticuloendothelial system. International Journal of Pharmaceutics 2000;202:1-10.

[159] McAllister K, Sazani P, Adam M, Cho MJ, Rubinstein M, Samulski RJ, et al. Polymeric Nanogels Produced via Inverse Microemulsion Polymerization as Potential Gene and Antisense Delivery Agents. J. Amer. Chem. Soc. 2002;124:15198-207.

[160] Craparo EF, Cavallaro G, Bondi ML, Mandracchia D, Giammona G. PEGylated Nanoparticles Based on a Polyaspartamide. Preparation, Physico-Chemical Characterization, and Intracellular Uptake. Biomacromolecules 2006;7:3083-92.

[161] Oh JK, Tang C, Gao H, Tsarevsky NV, Matyjaszewski K. Inverse Miniemulsion ATRP: A New Method for Synthesis and Functionalization of Well-Defined Water-Soluble/Cross-Linked Polymeric Particles. J. Am. Chem. Soc. FIELD Full Journal Title:Journal of the American Chemical Society 2006;128:5578-84.

[162] Oh JK, Siegwart DJ, Lee H-i, Sherwood G, Peteanu L, Hollinger JO, et al. Biodegradable Nanogels Prepared by Atom Transfer Radical Polymerization as Potential Drug Delivery 
Carriers: Synthesis, Biodegradation, in Vitro Release, and Bioconjugation. J. Am. Chem. Soc. FIELD Full Journal Title:Journal of the American Chemical Society 2007;129:5939-45.

[163] Oh JK, Siegwart DJ, Matyjaszewski K. Synthesis and Biodegradation of Nanogels as Delivery Carriers for Carbohydrate Drugs. Biomacromolecules 2007;8:3326-31.

[164] Siegwart DJ, Oh JK, Gao H, Bencherif SA, Perineau F, Bohaty AK, et al. Biotin-, pyrene-, and GRGDS-functionalized polymers and nanogels via ATRP and end group modification. Macromol. Chem. Phys. 2008;209:2179-93.

[165] Siegwart DJ, Srinivasan A, Bencherif SA, Karunanidhi A, Oh JK, Vaidya S, et al. Cellular Uptake of Functional Nanogels Prepared by Inverse Miniemulsion ATRP with Encapsulated Proteins, Carbohydrates, and Gold Nanoparticles. Biomacromolecules 2009;10:2300-9.

[166] Bencherif SA, Siegwart DJ, Srinivasan A, Horkay F, Hollinger JO, Washburn NR, et al. Nanostructured hybrid hydrogels prepared by a combination of atom transfer radical polymerization and free radical polymerization. Biomaterials 2009;30:5270-8.

[167] Bencherif SA, Washburn NR, Matyjaszewski K. Synthesis by AGET ATRP of Degradable Nanogel Precursors for In Situ Formation of Nanostructured Hyaluronic Acid Hydrogel. Biomacromolecules 2009;10:2499-507.

[168] Oh JK, Perineau F, Matyjaszewski K. Preparation of Nanoparticles of Well-Controlled WaterSoluble Homopolymers and Block Copolymers Using an Inverse Miniemulsion ATRP. Macromolecules 2006;39:8003-10.

[169] Oh JK, Bencherif SA, Matyjaszewski K. Atom transfer radical polymerization in inverse miniemulsion: A versatile route toward preparation and functionalization of microgels/nanogels for targeted drug delivery applications. Polymer 2009;50:4407-23.

[170] Landfester K, Ramirez LP. Encapsulated magnetite particles for biomedical application. J. Phys. Condens. Matter 2003;15:S1345-S61.

[171] Xu ZZ, Wang CC, Yang WL, Deng YH, Fu SK. Encapsulation of nanosized magnetic iron oxide by polyacrylamide via inverse miniemulsion polymerization. Journal of Magnetism and Magnetic Materials 2004;277:136-43.

[172] Dresco PA, Zaitsev VS, Gambino RJ, Chu B. Langmuir 1999;15:1945.

[173] Wormuth K. Superparamagnetic Latex via Inverse Emulsion Polymerization. Journal of Colloid and Interface Science 2001;241:366-77.

[174] Deng Y, Wang L, Yang W, Fu S, Elaissari A. Preparation of magnetic polymeric particles via inverse microemulsion polymerization process. Journal of Magnetism and Magnetic Materials 2003;257:69-78.

[175] Arshady R. Suspension, emulsion, and dispersion polymerization: a methodological survey. Colloid Polym. Sci. 1992;270:717-32.

[176] Cawse JL, in Emulsion Polymerization and Emulsion Polymers (Ed.: P. Lovell, El-Aasser, M. S.), John Wiley \& Sons Ltd, West Sussex, England, 1997, p. 743.

[177] Song J-S, Tronc F, Winnik MA. Two-Stage Dispersion Polymerization toward Monodisperse, Controlled Micrometer-Sized Copolymer Particles. J. Amer. Chem. Soc. 2004;126:6562-3.

[178] Horak D, Bohacek J, Subrt M. Magnetic poly(2-hydroxyethyl methacrylate-co-ethylene dimethacrylate) microspheres by dispersion polymerization. J. Polym. Sci. Part A Polym. Chem. 2000;38:1161-71.

[179] Horak D. Magnetic poly(glycidyl methacrylate) microspheres by dispersion polymerization. J. Polym. Sci. Part A Polym. Chem. 2001;39:3707-15.

[180] Horak D, Petrovsky E, Kapicka A, Frederichs T. Synthesis and characterization of magnetic poly(glycidyl methacrylate) microspheres. Journal of Magnetism and Magnetic Materials 2007;311:500-6. 
[181] Horak D, Lednicky F, Petrovsky E, Kapicka A. Magnetic characteristics of ferrimagnetic microspheres prepared by dispersion polymerization. Macromol. Mater. Eng. 2004;289:341-8.

[182] Mackova H, Kralova D, Horak D. Magnetic poly(N-isopropylacrylamide) microspheres by dispersion and inverse emulsion polymerization. J. Polym. Sci., Part A: Polym. Chem. 2007;45:5884-98.

[183] Zhang J, Yu D, Chen W, Xie Y, Wan W, Liang H, et al. Preparation of poly(styrene-glycidyl methacrylate)/Fe3O4 composite microspheres with high magnetite contents. Journal of Magnetism and Magnetic Materials 2009;321:572-7.

[184] Purushotham S, Chang PEJ, Rumpel H, Kee IHC, Ng RTH, Chow PKH, et al. Thermoresponsive core-shell magnetic nanoparticles for combined modalities of cancer therapy. Nanotechnology 2009;20:305101.

[185] Zhang J, Xu S, Kumacheva E. Polymer Microgels: Reactors for Semiconductor, Metal, and Magnetic Nanoparticles. J. Amer. Chem. Soc. 2004;126:7908-14.

[186] Pich A, Bhattacharya S, Lu Y, Boyko V, Adler H-JP. Temperature-Sensitive Hybrid Microgels with Magnetic Properties. Langmuir 2004;20:10706-11.

[187] Dong H, Mantha V, Matyjaszewski K. Thermally Responsive PM(EO)2MA Magnetic Microgels via Activators Generated by Electron Transfer Atom Transfer Radical Polymerization in Miniemulsion. Chemistry of Materials 2009;21:3965-72.

[188] Mohammadi Z, Wang X, Berkland C. Magnetic polyvinylamine nanoparticles by in situ precipitation reaction. J. Polym. Sci. Part A Polym. Chem. 2010;48:991-6.

[189] Liu T-Y, Hu S-H, Liu K-H, Liu D-M, Chen S-Y. Study on controlled drug permeation of magnetic-sensitive ferrogels: Effect of Fe3O4 and PVA. J. Controlled Release 2008;126:228-36.

[190] Liu T-Y, Hu S-H, Liu T-Y, Liu D-M, Chen S-Y. Magnetic-Sensitive Behavior of Intelligent Ferrogels for Controlled Release of Drug. Langmuir 2006;22:5974-8.

[191] De Paoli VM, Lacerda SHDP, Spinu L, Ingber B, Rosenzweig Z, Rosenzweig N. Effect of an Oscillating Magnetic Field on the Release Properties of Magnetic Collagen Gels. Langmuir 2006;22:5894-9.

[192] Hernandez R, Mijangos C. In situ synthesis of magnetic iron oxide nanoparticles in thermally responsive alginate-poly( $\mathrm{N}$-isopropylacrylamide) semi-interpenetrating polymer networks. Macrom. Rapid Commun. 2009;30:176-81.

[193] Xulu PM, Filipcsei G, Zrinyi M. Preparation and Responsive Properties of Magnetically Soft Poly(N-isopropylacrylamide) Gels. Macromolecules 2000;33:1716-9.

[194] Babic M, Horak D, Jendelova P, Glogarova K, Herynek V, Trchova M, et al. Poly(N,Ndimethylacrylamide)-Coated Maghemite Nanoparticles for Stem Cell Labeling. Bioconjugate Chem. 2009;20:283-94.

[195] Horak D, Babic M, Jendelova P, Herynek V, Trchova M, Likavcanova K, et al. Effect of different magnetic nanoparticle coatings on the efficiency of stem cell labeling. Journal of Magnetism and Magnetic Materials 2009;321:1539-47.

[196] Byrne JD, Betancourt T, Brannon-Peppas L. Active targeting schemes for nanoparticle systems in cancer therapeutics. Adv. Drug Delivery Rev. 2008;60:1615-26.

[197] Khandare J, Minko T. Polymer-drug conjugates: Progress in polymeric prodrugs. Progress in Polymer Science 2006;31:359-97.

[198] Nishiyama N, Kataoka K. Nanostructured devices based on block copolymer assemblies for drug delivery: designing structures for enhanced drug function. Advances in Polymer Science 2006;193:67-101.

[199] Wu J, Eisenberg A. Proton Diffusion across Membranes of Vesicles of Poly(styrene-b-acrylic Acid) Diblock Copolymers. J. Amer. Chem. Soc. 2006;128:2880-4. 
[200] Tomalia DA. Birth of a new macromolecular architecture: dendrimers as quantized building blocks for nanoscale synthetic polymer chemistry. Progress in Polymer Science 2005;30:294324.

[201] Farokhzad OC, Cheng J, Teply BA, Sherifi I, Jon S, Kantoff PW, et al. Targeted nanoparticleaptamer bioconjugates for cancer chemotherapy in vivo. Proc. Nat. Acad. Sci. USA 2006; 103:6315-20.

[202] Oh JK, Lee DI, Park JM. Biopolymer-based microgels/nanogels for drug delivery applications. Progress in Polymer Science 2009;34:1261-82.

[203] Peppas NA, Hilt JZ, Khademhosseini A, Langer R. Hydrogels in biology and medicine: from molecular principles to bionanotechnology. Advanced Materials 2006;18:1345-60.

[204] Hamidi M, Azadi A, Rafiei P. Hydrogel nanoparticles in drug delivery. Adv. Drug Delivery Rev. 2008;60:1638-49.

[205] Oh JK. Engineering of nanometer-sized cross-linked hydrogels for biomedical applications. Can. J. Chem. 2010;88:173-84.

[206] Lattermann G, Krekhova M. Thermoreversible ferrogels. Macromol. Rapid Commun. 2006;27:1373-9.

[207] Qin J, Asempah I, Laurent S, Fornara A, Muller RN, Muhammed M. Advanced Materials 2009;21:1354-7.

[208] Gupta AK, Curtis ASG. Surface modified superparamagnetic nanoparticles for drug delivery: Interaction studies with human fibroblasts in culture. J. Mater. Sci. Mater. Med. 2004;15:493-6.

[209] Dong H, Mantha V, Matyjaszewski K. Chemistry of Materials 2009;21:3965-72.

[210] Gonzales-Weimuller M, Zeisberger M, Krishnan KM. Size-dependant heating rates of iron oxide nanoparticles for magnetic fluid hyperthermia. Journal of Magnetism and Magnetic Materials 2009;321:1947-50.

[211] Kim D-H, Kim K-N, Kim K-M, Lee Y-K. Targeting to carcinoma cells with chitosan- and starch-coated magnetic nanoparticles for magnetic hyperthermia. J. Biomed. Mater. Res. Part A 2008;88A:1-11.

[212] Meenach SA, Anderson AA, Suthar M, Anderson KW, Hilt JZ. Biocompatibility analysis of magnetic hydrogel nanocomposites based on poly(N-isopropylacrylamide) and iron oxide. J. Biomed. Mater. Res. Part A 2009;91A:903-9.

[213] Hong J, Xu D, Yu J, Gong P, Ma H, Yao S. Facile synthesis of polymer-enveloped ultrasmall superparamagnetic iron oxide for magnetic resonance imaging. Nanotechnology 2007;18:135608/1-/6.

[214] Hiergeist R, Andra W, Buske N, Hergt R, Hilger I, Richter U, et al. Application of magnetite ferrofluids for hyperthermia. Journal of Magnetism and Magnetic Materials 1999;201:420-2.

[215] Le Renard P-E, Jordan O, Faes A, Petri-Fink A, Hofmann H, Ruefenacht D, et al. The in vivo performance of magnetic particle-loaded injectable, in situ gelling, carriers for the delivery of local hyperthermia. Biomaterials 31:691-705.

[216] Huang J, Han B, Yue W, Yan H. Magnetic polymer microspheres with polymer brushes and the immobilization of protein on the brushes. Journal of Materials Chemistry 2007;17:3812-8. 